\title{
Velocity-Aware Handover Management in Two-Tier Cellular Networks
}

\author{
Rabe Arshad, Hesham ElSawy, Sameh Sorour, Tareq Y. Al-Naffouri, and Mohamed-Slim Alouini
}

\begin{abstract}
While network densification is considered an important solution to cater the ever-increasing capacity demand, its effect on the handover (HO) rate is overlooked. In dense 5G networks, HO delays may neutralize or even negate the gains offered by network densification. Hence, user mobility imposes a nontrivial challenge to harvest capacity gains via network densification. In this paper, we propose a velocityaware HO management scheme for two-tier downlink cellular network to mitigate the $\mathrm{HO}$ effect on the foreseen densification throughput gains. The proposed HO scheme sacrifices the best base station (BS) connectivity, by skipping HO to some BSs along the user trajectory, to maintain longer connection durations and reduce HO rates. Furthermore, the proposed scheme enables cooperative BS service and strongest interference cancellation to compensate for skipping the best connectivity. To this end, we consider different HO skipping scenarios and develop a velocityaware mathematical model, via stochastic geometry, to quantify the performance of the proposed $\mathrm{HO}$ schemes in terms of the coverage probability and user throughput. The results highlight the HO rate problem in dense cellular environments and show the importance of the proposed HO schemes. Finally, the value of BS cooperation along with handover skipping is quantified for different user mobility profiles.
\end{abstract}

Index Terms-Multi-tier Dense Cellular Networks; Handover Management; Stochastic Geometry; CoMP; Throughput.

\section{INTRODUCTION}

$\mathbf{N}$ ETWORK densification is a potential solution to cater the increasing traffic demands and is expected to have a major contribution in fulfilling the ambitious 1000-fold capacity improvements required for next generation $5 \mathrm{G}$ cellular networks [1]. Network densification improves the spatial frequency reuse by shrinking the base stations' (BS) footprints to increase the delivered spatial spectral efficiency. Densifying the network decreases the load served by each BS, and hence, increases the per user throughput. However, such improvement comes at the expense of increased handover (HO) rates for mobile users. Mobile users change their BS associations more frequently in a denser network environment, due to the reduced BSs' footprints, to maintain the best connectivity. The HO procedure involves signaling between the mobile user, serving $\mathrm{BS}$, target BS, and the core network, which consumes physical

Rabe Arshad is with the Department of Electrical Engineering, King Fahd University of Petroleum and Minerals, Dhahran, 31261, Saudi Arabia. E-mail: g201408420@kfupm.edu.sa.

Hesham Elsawy, Tareq Y. Al-Naffouri and Mohamed-Slim Alouini are with the CEMSE Division, EE Program, King Abdullah University of Science and Technology, Thuwal, 23955-6900, Saudi Arabia. Email: \{hesham.elsawy, tareq.alnaffouri, slim.alouini\} @ kaust.edu.sa.

Sameh Sorour is with the Department of Electrical and Computer Engineering, University of Idaho, Moscow, 83844, USA. E-mail: samehsorour@uidaho.edu. resources and incurs delay. Therefore, the per user $\mathrm{HO}$ rate is always a performance limiting parameter for cellular operators. In modern cellular environments, high mobility and dense deployment of BSs are not mutually exclusive (e.g. monorails in downtowns), which may lead to service failures due to small dwell times within each BS footprint. Such problem will become more prominent in the foreseen ultra-dense cellular networks [2], [3].

The HO rate is an important key performance indicator (KPI) for cellular operators, thus minimizing HO delay and unnecessary HOs is a focal research point in the context of mobility management [4]-[7]. The authors in [4] present an handover management algorithm based on Ant Colony to reduce unnecessary HOs and radio link failures in femto cellular networks. Another handover management algorithm to avoid unnecessary HOs is presented in [5] for a typical two tier cellular network and in [6] for cloud-RAN based multitier networks. A HO management technique, based on self organizing maps is proposed in [7] to reduce unnecessary HOs for indoor users in two tier cellular networks. However, none of the aforementioned references study the interplay between HO delay and BS intensity or the effect of HO delay on the user throughput.

Motivated by the importance of network densification and the significance of the $\mathrm{HO}$ problem, several researchers started to exploit stochastic geometry to characterize, understand, and solve the HO problem in dense cellular networks. Stochastic geometry is a powerful mathematical tool that has shown success to characterize the performance of cellular networks with stationary users [8], [9]. Using stochastic geometry, the handover rate in cellular networks is characterized in [10] for a single tier cellular network with the random waypoint mobility model and in [11] for a multi-tier cellular network with an arbitrary mobility model. However, [10] and [11] focus only on the $\mathrm{HO}$ rate and do not investigate the effect of HO on the throughput. Stochastic geometry models that incorporate handover effect into throughput analysis can be found in [12]-[14]. However, none of [12]-[14] propose a solution to the excessive HO rate problem. The authors in [15], propose control plane and user plane split architecture with macro BS anchoring to mitigate the handover effect in dense cellular environment and quantified the performance gain via stochastic geometry. However, the solution proposed in [15] is not compatible with the current cellular networks and requires massive architectural upgrade to the network.

In this paper, we propose a simple yet effective velocity aware handover management scheme in a two-tier cellular network that is compatible with the current cellular archi- 
tecture. ${ }^{1}$ The proposed scheme, denoted as HO skipping, bypasses association with some BSs along the user trajectory to maintain a longer service duration with serving BSs and reduce the $\mathrm{HO}$ rate and its associated signaling. In other words, the proposed HO skipping scheme sacrifices the best signalto-interference-plus-noise-ratio (SINR) association to alleviate excessive $\mathrm{HO}$ rate and mitigate the handover effect. The proposed scheme also employs interference cancellation (IC) and cooperative BS service, via coordinated multipoint (CoMP) transmission [18]-[20], when the user is not associated to the BS offering the best SINR. When the user decides to skip the best SINR association, denoted as blackout phase, the user is simultaneously served by the BSs that offer the second and third best SINR associations via non-coherent transmission. It is worth mentioning that the non-coherent transmission is considered as it may be hard to estimate the channel state information (CSI) in the considered high mobility scenarios.

The performance gain of the proposed HO skipping scheme is quantified using stochastic geometry, in which the cellular network is assumed to be spatially deployed according to a Poisson point process (PPP). The PPP assumption is widely accepted for modeling cellular networks and has been verified in [21]-[23] by several empirical studies. To this end, we derive mathematical expressions for the coverage probabilities and the average throughput for the proposed HO skipping schemes. The results manifest the HO problem in dense cellular environments when employing the conventional $\mathrm{HO}$ scheme (i.e., best SINR association). Compared to the always best SINR connectivity, the proposed HO schemes show some degradation in the overall coverage probability, but tangible gains are achieved in terms of average throughput.

The rest of the paper is organized as follows. In Section II, we present the system model and propose HO skipping strategies. Coverage probabilities, $\mathrm{HO}$ rates and average throughput are analyzed in Section III, IV, and V, respectively. Finally, the paper is concluded in Section VI.

\section{SySTEM MODEL}

We consider a two-tier downlink cellular network with CoMP transmission between BSs belonging to the same or different tiers. It is assumed that the BSs belonging to the $k^{\text {th }}$ tier have same transmit power $P_{k}$, bias factor ${ }^{2} B_{k}$, and are spatially distributed via a two-dimensional homogenous PPP $\Phi_{k}$ with intensity $\lambda_{k}, k \in\{1,2\}$. The macro and femto cell tiers are denoted by $k=1$ and $k=2$, respectively. A power-law path-loss model with path loss exponent $\eta>2$ is considered. For simplicity, we consider the same path loss exponents for the two tiers (i.e. $\eta_{1}=\eta_{2}=\eta$ ). Extensions to different path-loss exponents is straightforward, but comes at the expense of more involved expressions. In addition to path loss, the channel introduces multi-path fading, which is assumed to be Rayleigh distributed with unit power i.e. $h \sim \exp (1)$. Without loss of generality, we conduct our

\footnotetext{
${ }^{1}$ This work has been presented in parts for single tier cellular network in [16], [17].

${ }^{2}$ Biasing is used to manipulate user-to-BS association in order to fulfill a certain network wide objective such as traffic-offloading, load-balancing, and congestion relief [24]-[26].
}

analysis on a test user and assume that all BSs in $\Phi_{1}$ and $\Phi_{2}$ are ascendingly ordered according to their distances from that user. Let $R_{i}$ and $r_{i}$ be the distances from the test user to the $i^{\text {th }}$ BS in $\Phi_{1}$ and $\Phi_{2}$, respectively, then the inequalities $\left(R_{1}<R_{2}<\ldots\right)$ and $\left(r_{1}<r_{2}<\ldots\right)$ always hold. We consider a universal frequency reuse scheme and study the performance of one frequency channel. Hence, the best received signal strength (RSS) association implies the best SINR association. A list of key mathematical notations used in this paper is given in table I.

TABLE I: Mathematical Notations

\begin{tabular}{||c|c||}
\hline Notation & Description \\
\hline$\Phi_{k}$ & PPP of BSs of $k^{t h}$ tier \\
\hline$\eta$ & Path loss exponent \\
\hline$\lambda_{k}$ & Bransmit power of BSs of $k^{t h}$ tier \\
\hline$P_{k}$ & Bias factor of $k^{t h}$ tier \\
\hline$B_{k}$ & Distance between the user and $i^{t h}$ macro BS \\
\hline$R_{i}$ & Distance between the user and $i^{t h}$ femto BS \\
\hline$r_{i}$ & Achievable rate per unit bandwidth \\
\hline$H_{i j}$ & Macro to macro HO delay \\
\hline $\mathcal{R}$ & Femto related HO delay \\
\hline$d_{m}$ & Coverage probability \\
\hline$d_{f}$ & Average Throughput \\
\hline $\mathcal{C}$ & best SINR \\
\hline$A T$ & The probability that the macro BS provides the \\
\hline$A_{m}$ & best SINR \\
\hline$A_{f}$ & The probability that the femto BS provides the \\
\hline
\end{tabular}

\section{A. User Mobility and Handover Strategies}

In the depicted system model, the association regions for the BSs can be visualized via a weighted voronoi tessellation [27] (as shown in Fig. 1), which is widely considered in literature (e.g. [11], [15]). The conventional scheme executes a HO every time the user crosses a voronoi cell boundary to ensure that the best SINR association is always satisfied. We assume that the test user moves with a constant velocity $v$ on an arbitrary long trajectory ${ }^{3}$ that passes through all association and SINR states. The average SINR through a randomly selected user trajectory is inferred from the stationary PPP analysis. It is worth noting that a similar assumption was used in [12]-[15] for tractability. However, we incorporate user mobility in the simulations and verify the accuracy of the stationary SINR analysis for mobile users. This implies that averaging over all users' trajectories in all network realizations is equivalent to averaging over all users' locations in all network realizations.

We propose multiple HO skipping strategies that show throughput gains over different user mobility profiles. Particularly, we consider four HO strategies, which represent user mobility profiles ranging from nomadic to high velocities. Mobile users maintain a list of nearby BSs based on the RSS

\footnotetext{
${ }^{3}$ The handover analysis in this paper is based on the mathematical model developed in [11], which is valid for an arbitrary trajectory shape and mobility model.
} 


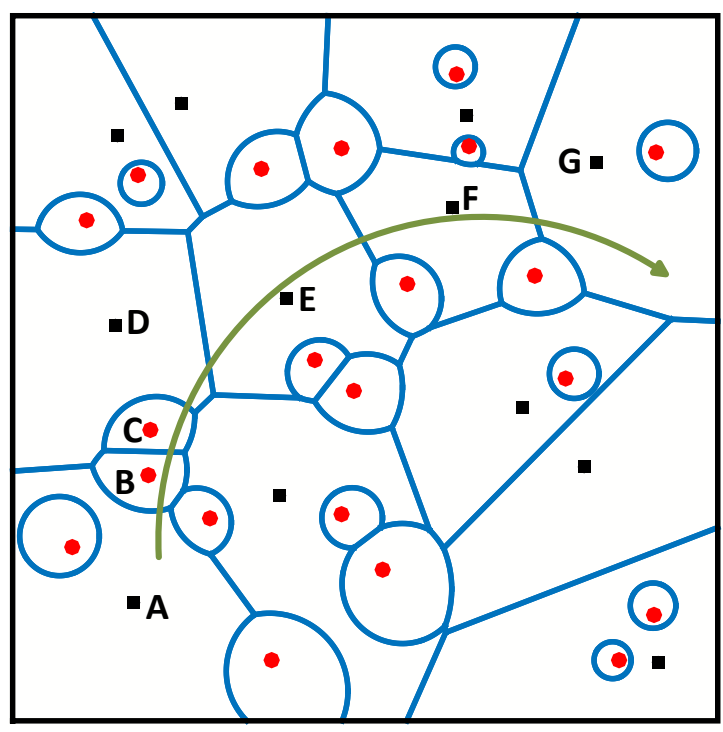

(a) Without biasing

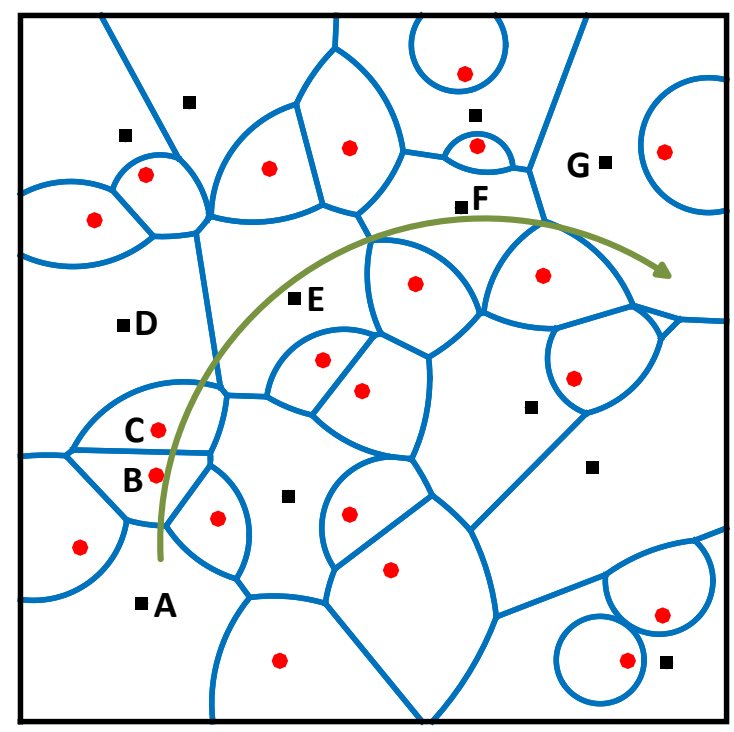

(b) With biasing

Fig. 1: Weighted Voronoi tessellations of two tier cellular network. Green solid line represents user trajectory while black squares and red circles represent macro and femto BSs, respectively.

levels and report to the core network through the serving BS. In some cases, HO decisions are made on the radio network level based on the HO type. However, in all cases, the HOs are directed by the network entities, which have the capabilities to track the user location and velocity [28]-[30]. According to the employed HO strategy, the admission controller can help users skip the recommended HOs based on their velocities. BS skipping sacrifices the best SINR connectivity to reduce the handover rate and delay. In order to compensate for the degraded SINR during blackout phases, we enable BS cooperation and IC. For the IC, the interfering signal from the skipped BS is detected, demodulated, decoded and then subtracted from the received signal [31].

In this paper, we propose the following HO strategies for the mobile users.

1) Best Connected Strategy (BC): In the best connected strategy, the admission controller ensures that the biased RSS based association is always satisfied for each $\mathrm{HO}$ request received from the mobile station. That is, the user is connected to the nearest macro $\mathrm{BS}$ if $P_{1} B_{1} R_{1}^{-\eta}>P_{2} B_{2} r_{1}^{-\eta}$ is satisfied and to the nearest femto $\mathrm{BS}$ if $P_{1} B_{1} R_{1}^{-\eta}<P_{2} B_{2} r_{1}^{-\eta}$ is satisfied. For the user trajectory shown in Fig. 1(a), the best connected strategy enforces $6 \mathrm{HOs}$ when the user moves from BS A to $G$ through BSs $\{B, C, D, E, F\}$.

2) Femto Skipping Strategy (FS): In the femto skipping strategy, we propose that the user skips some of the femto BSs along its trajectory, when $P_{1} B_{1} R_{1}^{-\eta}<P_{2} B_{2} r_{1}^{-\eta}$ is satisfied, to reduce the handover rate. In particular, the user can alternate between the best connectivity and skipping of the femto BSs along its trajectory. During the femto blackout phase, BS cooperation is enabled, which can be intra or intertier cooperative BS transmission depending on the relative positions of the BSs along the user trajectory. For the user trajectory shown in Fig. 1(a), the FS strategy involves 5 HOs (i.e., $\{\mathrm{C}, \mathrm{D}, \mathrm{E}, \mathrm{F}, \mathrm{G}\}$ ) in moving from BS A to G. Also, the user is jointly served by the BSs $\{$ A, C $\}$ while skipping BS B.

3) Femto Disregard Strategy (FD): At high mobility profiles, the cell dwell time within the femto BS coverage area may be too small. Hence, we propose the femto disregard strategy where the user skips HOs to the entire femto tier while enabling the cooperative service between the two strongest macros in blackout. This states that the user connects to the nearest macro BS if $P_{1} B_{1} R_{1}^{\eta}>P_{2} B_{2} r_{1}^{-\eta}$ and to the first and the second strongest macros, otherwise. For the user trajectory shown in Fig. 1(a), the FD strategy offers 4 HOs (i.e., $\{\mathrm{D}, \mathrm{E}, \mathrm{F}, \mathrm{G}\}$ ) and the joint transmission between the BSs $\{$ A, D $\}$ is enabled while skipping of the BSs $\{\mathrm{B}, \mathrm{C}\}$.

4) Macro Skipping Strategy (MS) : At extremely high velocities, the cell dwell time within the macro BS area may become too small. In this case, in addition to the femto disregard, the user may skip some macro BSs along its trajectory. Particularly, the user alternates between the macro best connectivity and macro blackout phases, where macro BS cooperation in enabled in the macro blackout phase. That is, the user spends $50 \%$ of the time in macro best connected mode and rest $50 \%$ in the macro blackout mode. For the user trajectory shown in Fig. 1(a), the MS strategy enforces only 2 HOs (i.e., $\{\mathrm{E}, \mathrm{G}\}$ ) and cooperation is enabled between the BSs $\{A, E\}$ and $\{\mathrm{E}, \mathrm{G}\}$ while skipping of the macro BSs D and $\mathrm{F}$, respectively.

It is worth noting that the velocity ranges for each of the aforementioned skipping scheme are selected offline since they are based on the underlying BS intensity and per-BS HO delay. On the contrary, the selection of the skipped/non-skipped BSs is executed in real-time, which is facilitated by the prolonged 
association time offered by the proposed skipping algorithm.

\section{B. Methodology of Analysis}

We assume that no data is transmitted during HO execution and that the $\mathrm{HO}$ duration is dedicated for exchanging control signaling between the serving BS, target BS, and the core network. We consider different backhauling schemes that impose different $\mathrm{HO}$ and signaling delays [32]. In all cases, the achievable rate is calculated over the time interval over which only data can be transmitted. For each of the aforementioned HO skipping strategies, we show the imposed tradeoff between coverage probability and throughput. For the sake of an organized presentation, we show the analysis for each HO strategy in a separate section. In the analysis of each strategy, we first derive the distance distribution between the user and its serving BS(s) as well as the Laplace transform (LT) of the aggregate interference PDF, which are then used to obtain the coverage probability and achievable rate. As discussed earlier, the coverage probabilities and achievable rates are obtained based on the stationary analysis and are verified via simulations.

\section{Distance Analysis And Coverage Probability}

In this section, we first calculate the service distance distributions for the aforementioned HO skipping cases, which are subsequently used to obtain the coverage probabilities in each case. Note that the service distance distribution for each HO skipping case is different due to different serving BS(s) in each case. For the sake of an organized presentation, we perform case by case analysis. At the end of this section, we validate the stationary analysis via simulations that account for user mobility for all HO skipping scenarios.

\section{A. Best Connected Strategy (BC)}

In the best connectivity case, the user associates with the BS that provides the highest power. Thus the user changes its association when it crosses the boundary of the neighboring cell. The always best connected case has been considerably analyzed in the literature. Here, we follow [25] and write the distribution of the distances between the user and its serving macro and femto BSs in a two tier network, which is given by the following lemma.

Lemma 1 (Distance distributions in $\underline{B C}$ case): In a two tier cellular network, the distance distribution between the user and its serving macro BS is given by

$$
f_{R_{1}}^{(B C)}(R)=\frac{2 \pi \lambda_{1} R}{A_{m}^{(B C)}} \exp \left(-\pi R^{2}\left(\lambda_{1}+\lambda_{2}\left(\frac{\beta_{21} P_{2}}{P_{1}}\right)^{2 / \eta}\right)\right) ;
$$

where $0 \leq R \leq \infty$. The distance distribution between the user and its serving femto BS can be expressed as

$$
f_{r_{1}}^{(B C)}(r)=\frac{2 \pi \lambda_{2} r}{A_{f}^{(B C)}} \exp \left(-\pi r^{2}\left(\lambda_{2}+\lambda_{1}\left(\frac{\beta_{12} P_{1}}{P_{2}}\right)^{2 / \eta}\right)\right)
$$

where $0 \leq r \leq \infty$, and $\beta_{12}=\frac{B_{1}}{B_{2}}=\frac{1}{\beta_{21}}$ while $A_{m}^{(B C)}$ and $A_{f}^{(B C)}$ are the association probabilities for macro and femto BSs, respectively.

$$
A_{m}^{(B C)}=\frac{\lambda_{1}}{\lambda_{1}+\lambda_{2}\left(\frac{\beta_{21} P_{2}}{P_{1}}\right)^{2 / \eta}}, \quad A_{f}^{(B C)}=\frac{\lambda_{2}}{\lambda_{2}+\lambda_{1}\left(\frac{\beta_{12} P_{1}}{P_{2}}\right)^{2 / \eta}} .
$$

Proof: The lemma is obtained by using the same methodology as shown in [25, Lemma 3] but considering same path loss exponent for both tiers.

Coverage Probability: The coverage probability is defined as the probability that the received SINR exceeds a certain threshold $T$. In $\underline{B C}$ case, the user associates with the macro BS with probability $A_{m}^{(B C)}$ and with the femto BS with probability $A_{f}^{(B C)}$, where the association is based on the highest RSS. By the law of total probability, the overall coverage probability is given by

$$
\mathcal{C}^{(B C)}=A_{m}^{(B C)} \mathcal{C}_{m}^{(B C)}+A_{f}^{(B C)} \mathcal{C}_{f}^{(B C)},
$$

where $\mathcal{C}_{m}^{(B C)}$ and $\mathcal{C}_{f}^{(B C)}$ are the coverage probabilities for the serving macro and femto BSs, respectively. The coverage probabilities $\mathcal{C}_{m}^{(B C)}$ and $\mathcal{C}_{f}^{(B C)}$ are given by:

$$
\begin{aligned}
& \mathcal{C}_{m}^{(B C)}=\mathbb{P}\left[\frac{P_{1} h R_{1}^{-\eta}}{I_{R(m)}+I_{r(m)}+\sigma^{2}}>T\right], \\
& \mathcal{C}_{f}^{(B C)}=\mathbb{P}\left[\frac{P_{2} h r_{1}^{-\eta}}{I_{R(f)}+I_{r(f)}+\sigma^{2}}>T\right],
\end{aligned}
$$

where $I_{R(\cdot)}$ and $I_{r(\cdot)}$ are the aggregate interference powers received from the macro and femto tiers, respectively, which are defined as

$$
\begin{gathered}
I_{R(m)}=\sum_{i \epsilon \phi_{1} \backslash b_{1}} P_{1} h_{i} R_{i}^{-\eta}, \quad I_{r(m)}=\sum_{i \in \phi_{2}} P_{2} h_{i} r_{i}^{-\eta}, \\
I_{R(f)}=\sum_{i \in \phi_{1}} P_{1} h_{i} R_{i}^{-\eta}, \quad I_{r(f)}=\sum_{i \epsilon \phi_{2} \backslash b_{1}} P_{2} h_{i} r_{i}^{-\eta} .
\end{gathered}
$$

Following [8], conditioning on the distance between the user and the serving BS and exploiting the exponential distribution of $h_{i}$, the conditional coverage probabilities are given by

$$
\begin{aligned}
& \mathcal{C}_{m}^{(B C)}\left(R_{1}\right)=\exp \left(\frac{-T R_{1}^{\eta} \sigma^{2}}{P_{1}}\right) \mathscr{L}_{I_{R(m)}}\left(\frac{T R_{1}^{\eta}}{P_{1}}\right) \mathscr{L}_{I_{r(m)}}\left(\frac{T R_{1}^{\eta}}{P_{1}}\right), \\
& \mathcal{C}_{f}^{(B C)}\left(r_{1}\right)=\exp \left(\frac{-T r_{1}^{\eta} \sigma^{2}}{P_{2}}\right) \mathscr{L}_{I_{R(f)}}\left(\frac{T r_{1}^{\eta}}{P_{2}}\right) \mathscr{L}_{I_{r(f)}}\left(\frac{T r_{1}^{\eta}}{P_{2}}\right),
\end{aligned}
$$

where $\mathcal{C}_{m}^{(B C)}\left(R_{1}\right)$ and $\mathcal{C}_{f}^{(B C)}\left(r_{1}\right)$ are the conditional coverage probabilities for macro and femto associations, respectively. The LTs of $I_{R}$ and $I_{r}$ for the macro and femto association cases are evaluated in the following lemma.

Lemma 2 (LTs of the interference in BC case): The Laplace transforms of $I_{R}$ and $I_{r}$ in the macro association case are given by

$$
\mathscr{L}_{I_{R(m)}}(s)=\exp \left(-\frac{2 \pi \lambda_{1} T R_{1}^{2}}{\eta-2}{ }_{2} F_{1}\left(1,1-\frac{2}{\eta}, 2-\frac{2}{\eta},-T\right)\right),
$$

$$
\begin{aligned}
\mathscr{L}_{I_{r(m)}}(s)=\exp \left(-\frac{2 \pi \lambda_{2} T R_{1}^{2}}{\eta-2}\left(\frac{P_{2}}{P_{1}}\right)^{2 / \eta} \beta_{12}^{\eta-2 / \eta} .\right. \\
\left.{ }_{2} F_{1}\left(1,1-\frac{2}{\eta}, 2-\frac{2}{\eta},-T \beta_{12}\right)\right),
\end{aligned}
$$


The Laplace transforms of $I_{R}$ and $I_{r}$ in the femto association case can be expressed as

$$
\begin{aligned}
& \mathscr{L}_{I_{R(f)}}(s)=\exp \left(-\frac{2 \pi \lambda_{1} T r_{1}^{2}}{\eta-2}\left(\frac{P_{1}}{P_{2}}\right)^{2 / \eta} \beta_{21}^{\eta-2 / \eta} .\right. \\
& \left.{ }_{2} F_{1}\left(1,1-\frac{2}{\eta}, 2-\frac{2}{\eta},-T \beta_{21}\right)\right), \quad(11) \\
& \mathscr{L}_{I_{r(f)}}(s)=\exp \left(-\frac{2 \pi \lambda_{2} T r_{1}^{2}}{\eta-2}{ }_{2} F_{1}\left(1,1-\frac{2}{\eta}, 2-\frac{2}{\eta},-T\right)\right),
\end{aligned}
$$

where ${ }_{2} F_{1}(., ., .,$.$) is the Gauss hypergeometric function [33].$

Proof: See Appendix A.

In the special case when $\beta_{12}=1$ and $\eta=4$, which is a common path loss exponent for outdoor environments, the LTs in (9)-(12) boil down to much simpler expressions as shown below.

$$
\begin{gathered}
\left.\mathscr{L}_{I_{R(m)}}(s)\right|_{\eta=4}=\exp \left(-\pi \lambda_{1} R_{1}^{2} \sqrt{T} \arctan (\sqrt{T})\right), \\
\left.\mathscr{L}_{I_{r(m)}}(s)\right|_{\eta=4}=\exp \left(-\pi \lambda_{2} R_{1}^{2} \sqrt{\frac{T P_{2}}{P_{1}}} \arctan (\sqrt{T})\right) . \\
\left.\mathscr{L}_{I_{R(f)}}(s)\right|_{\eta=4}=\exp \left(-\pi \lambda_{1} r_{1}^{2} \sqrt{\frac{T P_{1}}{P_{2}}} \arctan (\sqrt{T})\right), \\
\mathscr{L}_{I_{r(f)}}(s)_{\eta=4}=\exp \left(-\pi \lambda_{2} r_{1}^{2} \sqrt{T} \arctan (\sqrt{T})\right) .
\end{gathered}
$$

Combining Lemmas 1 and 2, the following theorem is obtained for the coverage probability.

Theorem 1 (Coverage probabilities in $\underline{B C}$ case):

Considering two independent PPPs based two tier cellular network with BS intensity $\lambda_{i}$ in a Rayleigh fading environment, the coverage probabilities for the macro and femto users are given by (17) and (18), respectively.

Proof: The theorem is proved by substituting the LTs obtained in Lemma 2 in the conditional coverage probability expressions given in (7) and (8) and then integrating over the service distance distributions provided by Lemma 1 .

In an interference limited environment with path loss exponent $\eta=4$ and $\beta_{12}=1$, the coverage probabilities in Theorem 1 simplify to the following closed form expressions.

$$
\mathcal{C}_{m}^{(F S)}=\mathcal{C}_{f}^{(B C)}=\frac{1}{1+\sqrt{T} \arctan (\sqrt{T})} .
$$

\section{B. Femto Skipping Strategy (FS)}

In the femto skipping case, the test user associates with the macro BS based on the highest RSS. However, the user skips some femto BS associations to reduce excessive HO rate, where the user experience blackout during femto skipping. In the blackout phase, the user is simultaneously served by the second and the third strongest BSs via non-coherent CoMP transmission. The cooperating BSs can be both macros, both femtos, or one macro and one femto. We assume that the user alternates between the femto best connected and femto blackout phases. The service distance distributions for the best connectivity associations (i.e., non-blackout) in FS scheme are similar to that of $\mathrm{BC}$ scheme given in (1) and (2) i.e. $f_{R_{1}}^{(F S)}=f_{R_{1}}^{(B C)}$ and $f_{r_{1}}^{(\overline{F S)}}=f_{r_{1}}^{(B C)}$. However, in the blackout case, the distance distributions are different and have to be derived for each pair of cooperating base stations (i.e. macro and macro, femto and femto, macro and femto). Furthermore, the coverage probability in the blackout case is different for each of the cooperating BSs case and the probability of each cooperation event should be calculated to obtain the total coverage probability. That said, it is cumbersome to derive the distance distributions and coverage probabilities while accounting for the cooperative BSs types via the conventional procedure used in the literature and shown in Section III-A. Instead, we follow [34] and exploit the mapping theorem to develop a unified analysis for all cooperation instances by mapping the two dimensional PPPs into an equivalent one dimensional non-homogenous PPP.

Lemma 3 (Total BS intensity by mapping theorem): The two point processes $\Phi_{1}$ and $\Phi_{2}$ seen from the test receiver perspective are statistically equivalent to a one dimensional non-homogeneous PPP with intensity

$$
\lambda(y)=\frac{2 \pi}{\eta}\left(\lambda_{1}\left(B_{1} P_{1}\right)^{2 / \eta}+\lambda_{2}\left(B_{2} P_{2}\right)^{2 / \eta}\right) y^{2 / \eta-1} .
$$

Proof: See Appendix B.

Using Lemma 3, we do not need to account for the cooperating BSs types and are able to derive a unified distance distribution and coverage probability expression that accounts for all cooperation instances. This is demonstrated in the following lemma.

Lemma 4 (Distance distributions in $\boldsymbol{F S}$ case): Let $x$ and $y$ be the distances between the user and the cooperating BSs. Conditioning on $x$, the conditional distance distribution of the skipped BS with distance $r_{1}$ from the user conditioned on the second nearest $\mathrm{BS}$ in the blackout case is given by

$$
f_{r(b k)}^{(F S)}\left(r_{1} \mid x\right)=\frac{2 r_{1}^{2 / \eta-1}}{\eta x^{2 / \eta}} ; \quad 0 \leq r_{1} \leq x \leq \infty
$$

and the joint distance distribution of $x$ and $y$ is given by

$$
f_{X, Y(b k)}^{(F S)}(x, y)=\frac{4}{\eta^{2}}\left(\pi \lambda_{t}\right)^{3} x^{4 / \eta-1} y^{2 / \eta-1} \exp \left(-\pi \lambda_{t} y^{2 / \eta}\right) ;
$$

where $0 \leq x \leq y \leq \infty$ and $\lambda_{t}$ is given by

$$
\lambda_{t}=\lambda_{1}\left(B_{1} P_{1}\right)^{2 / \eta}+\lambda_{2}\left(B_{2} P_{2}\right)^{2 / \eta} .
$$

Proof: See Appendix C.

Coverage Probability: By the law of total probability, the overall coverage probability for the $\underline{\mathrm{FS}}$ case is given by

$$
\mathcal{C}^{(F S)}=A_{m(\overline{b k})}^{(F S)} \mathcal{C}_{m(\overline{b k})}^{(F S)}+A_{f(\overline{b k})}^{(F S)} \mathcal{C}_{f(\bar{k} \bar{k})}^{(F S)}+A_{b k}^{(F S)} \mathcal{C}_{b k}^{(F S)}
$$

where $\overline{b k}$ and $b k$ represent the non-blackout and blackout phases, respectively. The coverage probabilities for the macro and femto associations in the non-blackout case are the same as the probabilities derived in (17) and (18) i.e., $\mathcal{C}_{m(\overline{b k})}^{(\bar{F})}=$ $\mathcal{C}_{m}^{(B C)}$ and $\mathcal{C}_{f(\bar{k})}^{(F S)}=\mathcal{C}_{f}^{(B C)}$. Also, the macro association probability $A_{m(b \bar{k})}^{(F S)}$ is the same as derived in Lemma 1. In the blackout phase, the user skips the strongest femto BS candidate and is served by the second and the third strongest BSs via non-coherent CoMP, which changes the blackout coverage probability and results in two femto association 


$$
\begin{array}{r}
\mathcal{C}_{m}^{(B C)}=\frac{2 \pi \lambda_{1}}{A_{m}^{(B C)}} \int_{0}^{\infty} R_{1} \exp \left\{-\frac{T R_{1}^{\eta} \sigma^{2}}{P_{1}}-\pi R_{1}^{2}\left(\lambda_{1}\left(1+\frac{2 T}{\eta-2}{ }_{2} F_{1}\left(1,1-\frac{2}{\eta}, 2-\frac{2}{\eta},-T\right)\right)+\lambda_{2}\left(\frac{\beta_{21} P_{2}}{P_{1}}\right)^{2 / \eta}\left(1+\frac{2 T \beta_{12}^{\eta}}{\eta-2}\right.\right.\right. \\
\left.\left.\left.{ }_{2} F_{1}\left(1,1-\frac{2}{\eta}, 2-\frac{2}{\eta},-T \beta_{12}\right)\right)\right)\right\} d R_{1} .
\end{array}
$$

$$
\begin{array}{r}
\mathcal{C}_{f}^{(B C)}=\frac{2 \pi \lambda_{2}}{A_{f}^{(B C)}} \int_{0}^{\infty} r_{1} \exp \left\{-\frac{T r_{1}^{\eta} \sigma^{2}}{P_{2}}-\pi r_{1}^{2}\left(\lambda_{2}\left(1+\frac{2 T}{\eta-2}{ }_{2} F_{1}\left(1,1-\frac{2}{\eta}, 2-\frac{2}{\eta},-T\right)\right)+\lambda_{1}\left(\frac{\beta_{12} P_{1}}{P_{2}}\right)^{2 / \eta}\left(1+\frac{2 T \beta_{21}^{\eta}}{\eta-2} .\right.\right.\right. \\
\left.\left.\left.{ }_{2} F_{1}\left(1,1-\frac{2}{\eta}, 2-\frac{2}{\eta},-T \beta_{21}\right)\right)\right)\right\} d r_{1} .
\end{array}
$$

probabilities (i.e., blackout and non-blackout associations). Since the user alternates between the femto best connected and femto blackout phases, the probabilities that the user is in femto best connected (non-blackout) and blackout phases can be expressed as $A_{f(b \bar{k})}^{(F S)}=A_{b k}^{(F S)}=0.5 A_{f}^{(B C)}$. By employing the mapping theorem given in Lemma 3 , we can lump the aggregate interference from both tiers and express the coverage probability in the blackout phase as

$$
\mathcal{C}_{X, Y(b k)}^{(B C)}=\mathbb{P}\left[\frac{\left|h_{1} x^{-\frac{1}{2}}+h_{2} y^{-\frac{1}{2}}\right|^{2}}{I_{a g g}+I_{r_{1}}+\sigma^{2}}>T\right],
$$

where

$$
I_{r_{1}}=\frac{h_{1}}{r_{1}}, \quad I_{a g g}=\sum_{i \in \phi \backslash b_{1}, b_{2}, b_{3}} \frac{h_{i}}{z_{i}},
$$

where $r_{1}$ represents the distance between the user and the skipped femto BS while $z_{i}$ represents the distance between the user and the interfering BSs belonging to both tiers. Since $h_{i}^{\prime} s$ are i.i.d. $\mathcal{C N}(0,1)$, such that $\left|x_{1} h_{1}+x_{2} h_{2}\right|^{2} \sim \exp \left(\frac{1}{x_{1}^{2}+x_{2}^{2}}\right)$, we can write the conditional coverage probability (conditioned on the serving BSs) as

$$
\begin{array}{r}
\mathcal{C}_{X, Y(b k)}^{(F S)}(x, y)=\exp \left(\frac{-T \sigma^{2}}{x^{-1}+y^{-1}}\right) \mathscr{L}_{I_{r_{1}}}\left(\frac{T}{x^{-1}+y^{-1}}\right) . \\
\mathscr{L}_{I_{a g g}}\left(\frac{T}{x^{-1}+y^{-1}}\right)
\end{array}
$$

The Laplace transforms of $I_{r_{1}}$ and $I_{a g g}$ are given by the following lemma.

Lemma 5 (LTs of the interference in FS case): The Laplace transform of $I_{r_{1}}$ can be expressed as

$$
\mathscr{L}_{I_{r_{1}}}(s)=\int_{0}^{x} \frac{2 r_{1}^{2 / \eta-1}}{\eta x^{2 / \eta}\left(1+\frac{T}{r_{1}\left(x^{-1}+y^{-1}\right)}\right)} d r_{1} .
$$

The Laplace transform of $I_{a g g}$ can be expressed as

$$
\begin{aligned}
\mathscr{L}_{I_{\text {agg }}}(s)=\exp \left(\frac{-2 \pi \lambda_{t} T y^{2 / n-1}}{(\eta-2)\left(x^{-1}+y^{-1}\right)} .\right. \\
\left.\quad{ }_{2} F_{1}\left(1,1-\frac{2}{\eta}, 2-\frac{2}{\eta}, \frac{-T}{x^{-1} y+1}\right)\right) .
\end{aligned}
$$

Proof: See Appendix D.

For the special case $(\eta=4)$, the LTs in (28) and (29) simplify to the expressions as given below.

$$
\left.\mathscr{L}_{I_{r_{1}}}(s)\right|_{\eta=4}=1-\sqrt{\frac{T y}{x+y}} \arctan \left(\sqrt{\frac{x+y}{T y}}\right)
$$

$$
\left.\mathscr{L}_{I_{a g g}}(s)\right|_{\eta=4}=\exp \left(-\pi \lambda_{t} \sqrt{\frac{T}{x^{-1}+y^{-1}}} \arctan \sqrt{\frac{T x}{x+y}}\right),
$$

Using the service distance distribution and the LTs derived in Lemma 4 and 5, the following theorem for the coverage probability is obtained.

\section{Theorem 2 (Coverage probability in FS case):}

Considering two independent PPPs based two tier downlink cellular network with BS intensity $\lambda_{i}$ in a Rayleigh fading environment, the coverage probability for the blackout users in FS case is given by (32).

Proof: The theorem is obtained by substituting the LTs shown in Lemma 5 in the conditional coverage probability expression (27) and integrating over the distance distribution obtained in Lemma 4.

Interference Cancellation: In the blackout phase, the interference from the skipped BS (i.e., $I_{r_{1}}$ ) may be overwhelming to the SINR. Hence, interference cancellation techniques could be employed to improve the coverage probability. By considering skipped BS interference cancellation, the coverage probability for the blackout user is given by the following theorem.

Theorem 3 (Coverage probability in FS case with IC): Considering an independent PPP based two-tier cellular network with BS intensity $\lambda_{i}$ in a Rayleigh fading environment, the coverage probability for blackout users with interference cancellation capabilities can be expressed as

Proof: The theorem is obtained using the same methodology for obtaining (32) but with eliminated $I_{r_{1}}$ from (27).

\section{Femto Disregard Strategy (FD)}

In FD case, the mobile user skips all femto BSs associations. Since the femto BS footprint is quite smaller than the macro BS footprint, we propose that the test user associates with the macro BSs only. More specifically, the user associates with the nearest macro $\mathrm{BS}$ if $P_{1} B_{1} R_{1}^{\eta}>P_{2} B_{2} r_{1}^{-\eta}$ is satisfied while cooperative $\mathrm{BS}$ service is invoked in blackout (i.e. $\left.P_{1} B_{1} R_{1}^{\eta}<P_{2} B_{2} r_{1}^{-\eta}\right)$. Since the femto tier is disregarded, only macro BS cooperation is allowed to compensate for the SINR degradation in blackout. In non-blackout case, the user associates with the macro BS offering highest RSS, thus, the distance distribution in this case is the same as in $\underline{\mathrm{BC}}$ case given in (1) i.e. $f_{R_{1}}^{(F D)}=f_{R_{1}}^{(B C)}$. However, the conditional 


$$
\mathcal{C}_{(b k)}^{(F S)}=\int_{0}^{\infty} \int_{x}^{\infty} \frac{8 \pi \lambda_{t}^{3} x^{2 / \eta-1} y^{2 / \eta-1}}{\eta^{3}} \exp \left\{-\pi y^{2 / \eta} \lambda_{t}-\frac{2 \pi \lambda_{t} T y^{2 / n-1}}{(\eta-2)\left(x^{-1}+y^{-1}\right)}{ }_{2} F_{1}\left(1,1-\frac{2}{\eta}, 2-\frac{2}{\eta}, \frac{-T}{x^{-1} y+1}\right)\right\} \int_{0}^{x} \frac{r_{1}^{2 / \eta-1}}{1+\frac{T r_{1}^{-1}}{x^{-1}+y^{-1}}} d r_{1} d y d x
$$

$$
\mathcal{C}_{(b k, I C)}^{(F S)}=\int_{0}^{\infty} \int_{x}^{\infty} \frac{4 \pi \lambda_{t}^{3} x^{4 / \eta-1} y^{2 / \eta-1}}{\eta^{2}} \exp \left\{-\pi y^{2 / \eta} \lambda_{t}-\frac{2 \pi \lambda_{t} T y^{2 / n-1}}{(\eta-2)\left(x^{-1}+y^{-1}\right)}{ }_{2} F_{1}\left(1,1-\frac{2}{\eta}, 2-\frac{2}{\eta}, \frac{-T}{x^{-1} y+1}\right)\right\} d y d x
$$

and the joint PDFs of the distances between the blackout user and its serving macros are given by the following lemma.

Lemma 6 (Distance distributions in FD case): The conditional distribution of the distance from the skipped femto $\mathrm{BS}$, conditioned on the distance from the serving macro BS, is given by

$$
f_{r}^{(F D)}\left(r_{1} \mid R_{1}\right)=\frac{2 \pi \lambda_{2} r_{1} \exp \left(-\pi \lambda_{2} r_{1}^{2}\right)}{1-\exp \left(-\pi \lambda_{2} R_{1}^{2}\left(\frac{\beta_{21} P_{2}}{P_{1}}\right)^{2 / \eta}\right)}
$$

where $0 \leq r_{1} \leq\left(\frac{\beta_{21} P_{2}}{P_{1}}\right)^{1 / \eta} R_{1} \leq \infty$. The joint distance distribution between the test user and its skipped and serving $\mathrm{BSs}$ in the cooperative blackout mode can be expressed as

$f_{R_{1}, R_{2}, r_{1}(b k)}^{(F D)}(x, y, z)=\frac{(2 \pi)^{3}}{A_{b k}^{(F D)}} \lambda_{1}{ }^{2} \lambda_{2} x y z \exp \left(-\pi\left(\lambda_{1} y^{2}+\lambda_{2} z^{2}\right)\right)$.

The marginal distribution of the distance between the user and its first and second strongest macro BSs in the cooperative blackout mode is given by

$$
\begin{aligned}
f_{R_{1}, R_{2}(b k)}^{(F D)}(x, y)= & \frac{\left(2 \pi \lambda_{1}\right)^{2}}{A_{b k}^{(F D)}} x y \exp \left(-\lambda_{1} \pi y^{2}\right) . \\
& \left(1-\exp \left(-\lambda_{2} \pi x^{2}\left(\frac{\beta_{21} P_{2}}{P_{1}}\right)^{2 / \eta}\right)\right),
\end{aligned}
$$

where

$$
A_{b k}^{(F D)}=A_{f}^{(B C)}=\frac{\lambda_{2}}{\lambda_{2}+\lambda_{1}\left(\beta_{12} P_{1} / P_{2}\right)^{2 / \eta}} .
$$

Proof: The conditional distribution $f_{r}^{(F D)}\left(r_{1} \mid R_{1}\right)$ is obtained by first writing the joint distribution of $r_{1}$ and $R_{1}$ (i.e., $f_{r, R}\left(r_{1}, R_{1}\right)=(2 \pi)^{2} \lambda_{1} \lambda_{2} e^{-\pi \lambda_{1} R_{1}^{2}-\pi \lambda_{2} r_{1}^{2}}$ ). Then dividing it by the marginal distribution of $R_{1}$, $\left(r_{1}\left(\frac{\beta_{12} P_{1}}{P_{2}}\right)^{1 / \eta}<R_{1}<\infty\right)$, we obtain $f_{r}\left(r_{1} \mid R_{1}\right)$. The joint distribution $f_{R_{1}, R_{2}, r_{1}(b k)}^{(F D)}(., .,$.$) is obtained by first writ-$ ing the conditional PDF of $R_{2}$ conditioning on $R_{1}$ as $f_{R_{2}}\left(y \mid R_{1}\right)=2 \pi \lambda y e^{-\lambda \pi\left(y^{2}-R_{1}^{2}\right)}$ and calculating the joint PDF $f_{R_{1}, R_{2}}(x, y)=f(y \mid x) f_{R_{1}}(x)$. Then, multiplying by the weighted distribution of $r_{1}$ (i.e., using null probability of PPP), we get the joint distribution as given in (35). The marginal distance distribution between the user and its serving BSs $f_{R_{1}, R_{2}(b k)}^{(F D)}(.,$.$) is obtained by integrating (35) with respect to$ $r_{1}$, which is bounded from 0 to $R_{1}\left(\frac{\beta_{21} P_{2}}{P_{1}}\right)^{1 / \eta}$.
Coverage Probability: By employing the law of total probability, the overall coverage probability in the FD scheme can be written as

$$
\mathcal{C}^{(F D)}=A_{b \bar{k}}^{(F D)} \mathcal{C}_{m(b \bar{k})}^{(F D)}+A_{b k}^{(F D)} \mathcal{C}_{m, m(b k)}^{(F D)}
$$

The event probabilities in the above equation are the same as in $\underline{\mathrm{BC}}$ case, given in (3) (i.e., $A_{\overline{b k}}^{(F D)}=A_{m}^{(B C)}$ and $A_{b k}^{(F D)}=$ $\left.A_{f}^{(B C)}\right)$. The coverage probability in the non-blackout case is the same as the macro association probability in $\mathrm{BC}$ case i.e. $\mathcal{C}_{m(F \bar{k})}^{(F D)}=\mathcal{C}_{m}^{(B C)}$. However, the coverage probability for the blackout case is different from the previous cases and is given by

$$
\mathcal{C}_{m, m(b k)}^{(F D)}=\mathbb{P}\left[\frac{\left|\sqrt{P_{1}} h_{1} R_{1}^{-\frac{\eta}{2}}+\sqrt{P_{1}} h_{2} R_{2}^{-\frac{\eta}{2}}\right|^{2}}{I_{R}+I_{r_{1}}+I_{r}+\sigma^{2}}>T\right] .
$$

where, $I_{R}$ and $I_{r}$ are the aggregate interference powers from the macro and femto tiers, respectively. $I_{r_{1}}$ is the interference power from the strongest femto BS. Here, it is worth noting that $I_{R}$ is the received aggregate interference from all macro BSs except $\left\{b_{1}, b_{2}\right\}$ and $I_{r}$ is the aggregate interference power received from all femtos except $\left\{b_{1}\right\}$. The conditional coverage probability (conditioned on the serving BSs) for the blackout case is given by

$$
\begin{array}{r}
\mathcal{C}_{m, m(b k)}^{(F D)}\left(R_{1}, R_{2}\right)=\exp \left(\frac{-T \sigma^{2}}{x_{1}^{2}+x_{2}^{2}}\right) \mathscr{L}_{I_{R}}\left(\frac{T}{x_{1}^{2}+x_{2}^{2}}\right) \mathscr{L}_{I_{r_{1}}}\left(\frac{T}{x_{1}^{2}+x_{2}^{2}}\right) \\
\mathscr{L}_{I_{r}}\left(\frac{T}{x_{1}^{2}+x_{2}^{2}}\right)
\end{array}
$$

where

$$
x_{i}=\sqrt{P_{i}} R_{i}^{-\eta / 2} \text { and } h_{i}^{\prime} s \text { are i.i.d. } \mathcal{C N}(0,1) .
$$

Since the user is in blackout, the condition $P_{2} B_{2} r_{1}^{-\eta}>$ $P_{1} B_{1} R_{1}^{-\eta}$ is satisfied. This implies that the first nearest femto BS must exist between 0 and $R_{1}\left(\frac{\beta_{21} P_{2}}{P_{1}}\right)^{1 / \eta}$. Therefore, we derive the LT of $I_{r}$ considering that the interfering femto BSs exist outside an interference exclusion circle with radius $r_{1}$ centered at the test receiver. The LTs of $I_{R}, I_{r_{1}}$ and $I_{r}$ in the blackout case are evaluated in the following lemma.

Lemma 7 (LTs of the interference in $\underline{F D}$ case): The LT of the aggregate interference power received from the macro tier in the blackout phase can be characterized as

$$
\begin{aligned}
\mathscr{L}_{I_{R}}(s)=\exp \left(\frac{-2 \pi T \lambda_{1} R_{2}^{2-\eta}}{(\eta-2)\left(R_{1}^{-\eta}+R_{2}^{-\eta}\right)} \cdot\right. \\
\left.\quad{ }_{2} F_{1}\left(1,1-\frac{2}{\eta}, 2-\frac{2}{\eta}, \frac{-T R_{2}^{-\eta}}{R_{1}^{-\eta}+R_{2}^{-\eta}}\right)\right) .
\end{aligned}
$$


The LT of $I_{r_{1}}$ can be expressed as

$$
\begin{aligned}
\mathscr{L}_{I_{r_{1}}}(s)=\frac{2 \pi \lambda_{2}}{1-e^{-\lambda_{2} \pi R_{1}^{2}\left(\frac{\beta_{21} P_{2}}{P_{1}}\right)^{2 / \eta}}} . \\
\int_{0}^{R_{1}\left(\frac{\beta_{21} P_{2}}{P_{1}}\right)^{1 / \eta}} \frac{r_{1} e^{-\pi \lambda_{2} r_{1}^{2}}}{\left(1+\frac{T P_{1}^{-1} P_{2} r_{1}^{-\eta}}{R_{1}^{-\eta}+R_{2}^{-\eta}}\right)} d r_{1} .
\end{aligned}
$$

The LT of the aggregate interference power received from the entire femto tier except $\left\{b_{1}\right\}$ is given by

$$
\begin{aligned}
\mathscr{L}_{I_{r}}(s)=\exp \left(\frac{-2 \pi \lambda_{2} P_{2} T}{(\eta-2) P_{1}\left(R_{1}^{-\eta}+R_{2}^{-\eta}\right)} r_{1}^{2-\eta} .\right. \\
\left.{ }_{2} F_{1}\left(1,1-\frac{2}{\eta}, 2-\frac{2}{\eta}, \frac{-P_{2} T r_{1}^{-\eta}}{P_{1}\left(R_{1}^{-\eta}+R_{2}^{-\eta}\right)}\right)\right) .
\end{aligned}
$$

Proof: The LT of $I_{R}$ is derived using the same procedure as shown for $\mathscr{L}_{I_{R}(m)}(s)$ in (9) but considering the macros aggregate interference from $R_{2}$ to $\infty$ and $s=\frac{T}{P_{1}\left(R_{1}^{-\eta}+R_{2}^{-\eta}\right)}$. Also, the LT of $I_{r_{1}}$ is derived in a similar way as eq. (8) in [16] but considering different $s$ (shown above) and the conditional service distribution shown in (34). The LT of $I_{r}$ is derived in a similar way as $\mathscr{L}_{I_{R}}(s)$ while taking the femto aggregate interference from $r_{1}$ to $\infty$.

The LTs in Lemma 7 for a special case at $\eta=4$, boil down to the closed form expressions as shown below.

$\left.\mathscr{L}_{I_{R}}(s)\right|_{\eta=4}=\exp \left(-\pi \lambda_{1} \sqrt{\frac{T}{R_{1}^{-4}+R_{2}^{-4}}} \arctan \left(\sqrt{\frac{T R_{1}^{4}}{R_{1}^{4}+R_{2}^{4}}}\right)\right)$.

$\left.\mathscr{L}_{I_{r}}(s)\right|_{\eta=4}=\exp \left(-\pi \lambda_{2} \sqrt{\frac{P_{2} P_{1}^{-1} T}{R_{1}^{-4}+R_{2}^{-4}}} \arctan \left(\sqrt{\frac{P_{2} P_{1}^{-1} T r_{1}^{-4}}{R_{1}^{-4}+R_{2}^{-4}}}\right)\right)$

Using the LTs and distance distributions found in Lemmas 6 and 7 , we obtain the final coverage probability for the blackout users in FD case as given in Theorem 4.

Theorem 4 (Coverage probability in $\underline{F D}$ case):

Considering two independent PPPs based two tier downlink cellular network with BS intensity $\lambda_{i}$ in a Rayleigh fading environment, the coverage probability for the blackout case is given in (46).

Proof: We obtain the coverage probability for the blackout user with cooperation by substituting the LTs found in Lemma 7 in the conditional coverage probability expression given in (40) and integrating it over the service distance distribution obtained in Lemma 6.

In the blackout phase, the user associates with the two strongest macro BSs while employing interference cancellation on the strongest femto BS. For the interference cancellation case, the blackout coverage probability is given by the following theorem.

Theorem 5 (Coverage probability in $\mathrm{FD}$ case with IC): Considering two independent PPPs based two tier downlink cellular network with BS intensity $\lambda_{i}$ in a Rayleigh fading environment, the coverage probability for blackout users in FD case with IC capabilities is expressed in (47).

Proof: The theorem is obtained using the same methodology for obtaining Theorem 4 but with eliminating $I_{r_{1}}$ from (40) and integrating over the joint distance distribution expressed in (35).

\section{Macro Skipping Strategy (MS)}

In $\underline{\text { MS }}$ scheme, the test user skips all femto BSs and every other macro BSs along its trajectory. Particularly, the user in this case alternates between the macro best connected and macro blackout modes. That is, in blackout phase, the test user skips the nearest macro BS and disregards the entire tier of femto BSs. Also, the cooperative non-coherent transmission from the second and the third nearest macro BSs is only activated during macro blackout. The conditional and joint service distance distributions for the test user in the blackout phase are given by the following lemma.

Lemma 8 (Distance distributions in MS case): The joint distance distribution between the user and its skipped and serving or cooperating BSs in the blackout mode is given by

$f_{R_{1}, R_{2}, R_{3}(b k)}^{(M S)}(x, y, z)=\left(2 \pi \lambda_{1}\right)^{3} x y z e^{-\pi \lambda_{1} z^{2}} ; 0 \leq x \leq y \leq z \leq \infty$

The joint PDF of the distances between the test user and its serving or cooperating BSs in the blackout phase with BS cooperation is given by

$$
f_{R_{2}, R_{3}(b k)}^{(M S)}(y, z)=4(\pi \lambda)^{3} y^{3} z e^{-\pi \lambda z^{2}} ; \quad 0 \leq y \leq z \leq \infty
$$

The conditional (i.e., conditioning on $R_{2}$ ) PDF of the distance between the test user and the skipped BS in the blackout case is given by

$$
f_{R(b k)}^{(M S)}\left(R_{1} \mid R_{2}\right)=\frac{2 R_{1}}{R_{2}^{2}} .
$$

The joint distance distribution between the user and the disregarded femto and serving macro BSs in the non-blackout mode is given by

$$
f_{R_{1}, r_{1}(b \bar{k})}^{(M S)}(x, y)=\frac{(2 \pi)^{2}}{A_{f}} \lambda_{1} \lambda_{2} x_{1} y_{1} \exp \left(-\pi \lambda_{1} x^{2}-\pi \lambda_{2} y^{2}\right),
$$

where $A_{f}$ is the probability that $P_{2} B_{2} r_{1}^{-\eta}>P_{1} B_{1} R_{1}^{-\eta}$, which is same as $A_{f}^{(B C)}$, given in (3). The marginal distribution of the distance between the user and its serving macro BS in non-blackout mode is given by

$$
\begin{aligned}
f_{R_{1}(\overline{b k})}^{(M S)}(x)= & \frac{2 \pi}{A_{f}} \lambda_{1} x\left(\exp \left(-\pi \lambda_{1} x^{2}\right)-\right. \\
& \left.\exp \left(-\pi x^{2}\left(\lambda_{1}+\lambda_{2}\left(\frac{\beta_{21} P_{2}}{P_{1}}\right)^{2 / \eta}\right)\right)\right) .
\end{aligned}
$$

Proof: The joint conditional distribution of $R_{1}$ and $R_{2}$ is the order statistics of two i.i.d. random variables with PDF $\frac{2 R}{R_{3}^{2}}$, where $0 \leq R \leq R_{3}$. The joint conditional distribution is given by $f_{R_{1}, R_{2}}\left(x, y \mid R_{3}\right)=\frac{8 x y}{R_{3}^{4}}$, where $0<x<y<R_{3}$. By following Bayes' theorem, the joint PDF $f_{R_{1}, R_{2}, R_{3}(b k)}^{(M S)}(. .,$. is obtained by multiplying the conditional joint PDF of $R_{1}$ and $R_{2}$ by the marginal PDF of $R_{3}$. The lemma follows by performing this marginalization over $R_{3}$, using its marginal distribution derived in eq. (2) in [35]. The joint PDF of $R_{2}$ and $R_{3}$ is obtained by integrating (48) w.r.t. $x$ from 0 to $y$. The conditional PDF is obtained by dividing the joint PDF 


$$
\begin{aligned}
& \mathcal{C}_{m, m(b k)}^{(F D)}=\int_{0}^{\infty} \int_{R_{1}}^{\infty} \int_{0}^{R_{1}\left(\frac{\beta_{21} P_{2}}{P_{1}}\right)^{1 / \eta}} \frac{(2 \pi)^{3} \lambda_{1}^{2} \lambda_{2} r_{1} R_{1} R_{2}}{\left(1+\frac{T P_{2} r_{1}^{-\eta}}{P_{1}\left(R_{1}^{-\eta}+R_{2}^{-\eta}\right)}\right) A_{b k}^{(F D)}} \exp \left(-\pi \lambda_{2} r_{1}^{2}-\pi \lambda_{1} R_{2}^{2}-\frac{T \sigma^{2}}{P_{1}\left(R_{1}^{-\eta}+R_{2}^{-\eta}\right)}-\right. \\
& \frac{2 \pi T}{(n-2)\left(R_{1}^{-\eta}+R_{2}^{-\eta}\right)}\left\{\lambda_{1} R_{2}^{2-\eta}{ }_{2} F_{1}\left(1,1-\frac{2}{\eta}, 2-\frac{2}{\eta},-\frac{T R_{2}^{-\eta}}{R_{1}^{-\eta}+R_{2}^{-\eta}}\right)+\frac{\lambda_{2} P_{2} r_{1}^{2-\eta}}{P_{1}} .\right. \\
& \left.\left.{ }_{2} F_{1}\left(1,1-\frac{2}{\eta}, 2-\frac{2}{\eta}, \frac{-P_{2} T r_{1}^{-\eta}}{P_{1}\left(R_{1}^{-\eta}+R_{2}^{-\eta}\right)}\right)\right\}\right) d r_{1} d R_{2} d R_{1} .
\end{aligned}
$$

$$
\begin{gathered}
\mathcal{C}_{m, m(b k, I C)}^{(F D)}=\int_{0}^{\infty} \int_{R_{1}}^{\infty} \int_{0}^{R_{1}\left(\frac{\beta_{21} P_{2}}{P_{1}}\right)^{1 / \eta}} \frac{(2 \pi)^{3}}{A_{b k}^{(F D)} \lambda_{1}{ }^{2} \lambda_{2} R_{1} R_{2} r_{1} \exp \left(-\pi\left(\lambda_{1} R_{2}^{2}+\lambda_{2} r_{1}^{2}\right)-\frac{2 \pi T}{(n-2)\left(R_{1}^{-\eta}+R_{2}^{-\eta}\right)} \cdot\right.} \\
\left.\quad\left\{\lambda_{1} R_{2}^{2-\eta} F_{1}\left(1,1-\frac{2}{\eta}, 2-\frac{2}{\eta},-\frac{T R_{2}^{-\eta}}{R_{1}^{-\eta}+R_{2}^{-\eta}}\right)+\frac{\lambda_{2} P_{2} r_{1}^{2-\eta}}{P_{1}}{ }_{2} F_{1}\left(1,1-\frac{2}{\eta}, 2-\frac{2}{\eta}, \frac{-P_{2} T r_{1}^{-\eta}}{P_{1}\left(R_{1}^{-\eta}+R_{2}^{-\eta}\right)}\right)\right\}\right) d r_{1} d R_{2} d R_{1} .
\end{gathered}
$$

in (48) by the marginal distribution in (49). The joint PDF $f_{R_{1}, r_{1}(\bar{k})}^{(M S)}(.,$.$) is obtained by using the null probability of$ independent PPPs and the marginal distribution in (52) is found by integrating (51) w.r.t. $y$ from 0 to $x\left(\frac{\beta_{21} P_{2}}{P_{1}}\right)^{1 / \eta}$.

Coverage Probability: By the law of total probability, we can write the overall coverage probability as

$$
\mathcal{C}^{(M S)}=A_{m(\overline{b k})}^{(M S)} \mathcal{C}_{m(\overline{b k})}^{(M S)}+A_{f(\overline{b k})}^{(M S)} \mathcal{C}_{m /(\overline{b k})}^{(M S)}+A_{b k}^{(M S)} \mathcal{C}_{m, m(b k)}^{(M S)}
$$

where $\mathcal{C}_{m(\bar{b})}^{(M S)}$ is the coverage probability for the best connected macro user while $\mathcal{C}_{m /(\bar{k})}^{(M S)}$ is the coverage probability for the macro user when the strongest femto candidate is disregarded (i.e., $\left.P_{2} B_{2} r_{1}^{-\eta}>P_{1} B_{1} R_{1}^{-\eta}\right)$. Also, $\mathcal{C}_{m, m(b k)}^{(M S)}$ is the blackout coverage probability as the user skips the nearest macro BS and associates with the second and the third strongest macro BSs. Since the test user skips every other macro BS, the user spends $50 \%$ of the time in association with the strongest macro and rest of the time in the blackout phase on average. Consequently, we assume $A_{b k}^{(M S)}$ to be 0.5 . Moreover, in the non-blackout case, the user is either in best connected mode (i.e. $P_{1} B_{1} R_{1}^{-\eta}>P_{2} B_{1} r_{1}^{-\eta}$ ) or it disregards the strongest femto and associates with the macro BS (i.e. $\left.P_{2} B_{2} r_{1}^{-\eta}>P_{1} B_{1} R_{1}^{-\eta}\right)$. Thus, $A_{m(\overline{b k})}^{(M S)}$ is considered to be $0.5 *\left(1-A_{f(\overline{b k})}\right)$, where $A_{f(\overline{b k})}=A_{f}^{(B C)}$, which is defined in (3). Also, $\mathcal{C}_{m(\overline{b k})}^{(M S)}$ is the same as the best connected coverage probability for macro association as expressed in $\underline{B C}$ case (i.e., $\left.\mathcal{C}_{m}^{(B C)}\right)$. However, $\mathcal{C}_{m \prime(\bar{k})}^{(M S)}$ can be written as

$$
\mathcal{C}_{m /(b \bar{k})}^{(M S)}=\mathbb{P}\left[\frac{P_{1} h_{1} R_{1}^{-\eta}}{I_{R}+I_{r_{1}}+I_{r}+\sigma^{2}}>T\right],
$$

where $I_{R}$ is the aggregate interference power from the entire macro tier except $\left\{b_{1}\right\}$ while $I_{r_{1}}$ is the interference power from the strongest femto $\mathrm{BS}$ which lies from 0 to $R_{1}\left(\frac{\beta_{21} P_{2}}{P_{1}}\right)^{1 / \eta}$ and $I_{r}$ is the aggregate interference power received from all femtos except $\left\{b_{1}\right\}$. The blackout coverage probability case can be expressed as

$$
\mathcal{C}_{m, m(b k)}^{(M S)}=\mathbb{P}\left[\frac{\left|\sqrt{P_{1}} h_{2} R_{2}^{-\eta / 2}+\sqrt{P_{1}} h_{3} R_{3}^{-\eta / 2}\right|^{2}}{I_{R_{1}}+I_{R}+I_{r}+\sigma^{2}}>T\right],
$$

where $I_{R_{1}}$ is the received interference power from the nearest skipped macro BS while $I_{R}$ is the aggregate interference power from the whole macro tier except $\left\{b_{1}, b_{2}, b_{3}\right\}$. Here, $I_{r}$ is the aggregate interference power received from the whole femto tier, which exists from 0 to $\infty$. We define $I_{R_{1}}$ and $I_{R}$ for the blackout case as

$$
I_{R_{1}}=P_{1} h_{1} R_{1}^{-\eta} \quad, \quad I_{R}=\sum_{i \epsilon \phi_{1} \backslash b_{1}, b_{2}, b_{3}} P_{1} h_{i} R_{i}^{-\eta} .
$$

Since $h_{i} \sim \exp (1)$, the conditional coverage probability for the non-blackout is given by

$$
\begin{array}{r}
\mathcal{C}_{m \prime(\bar{k})}^{(M S)}\left(R_{1}\right)=\exp \left(\frac{-T}{P_{1} R_{1}^{-\eta}}\right) \mathscr{L}_{I_{R}}\left(\frac{T}{P_{1} R_{1}^{-\eta}}\right) \mathscr{L}_{I_{r_{1}}}\left(\frac{T}{P_{1} R_{1}^{-\eta}}\right) . \\
\mathscr{L}_{I_{r}}\left(\frac{T}{P_{1} R_{1}^{-\eta}}\right),
\end{array}
$$

where the LTs of $I_{r_{1}}$ and $I_{r}$ are the same as given in (42) and (43), respectively, with the only difference that there is no $R_{2}$ in this case as the user is connected to one macro BS only. Also, $\mathscr{L}_{I_{R}}(s)$ is given in (9). Using the conditional coverage probability expression and the service distance distribution for the best connectivity associations (i.e., non-blackout), the following theorem is obtained for the coverage probability.

Theorem 6 (Coverage probability in MS case with FD): The coverage probability for macro association while disregarding the nearest femto BS in the non-blackout case is given by (57).

Proof: The theorem is proved by substituting the LTs in the conditional coverage probability expression (56) and integrating over the service distance distribution found in (52).

Since $h_{i}$ 's are i.i.d. $\mathcal{C N}(0,1)$ such that $\left|x_{2} h_{2}+x_{3} h_{3}\right|^{2} \sim$ $\exp \left(\frac{1}{x_{2}^{2}+x_{3}^{2}}\right)$, we can write the conditional coverage probability for the blackout user as

$$
\begin{aligned}
\mathcal{C}_{m, m(b k)}^{(M S)}\left(R_{2}, R_{3}\right)= & \exp \left(\frac{-T \sigma^{2}}{x_{2}^{2}+x_{3}^{2}}\right) \mathscr{L}_{I_{R_{1}}}\left(\frac{T}{x_{2}^{2}+x_{3}^{2}}\right) . \\
& \mathscr{L}_{I_{R}}\left(\frac{T}{x_{2}^{2}+x_{3}^{2}}\right) \mathscr{L}_{I_{r}}\left(\frac{T}{x_{2}^{2}+x_{3}^{2}}\right),
\end{aligned}
$$

where $x_{i}$ is the same as defined in FD case. Also, note that the LT of $I_{r}$ in the blackout case is different from the one expressed in FD case. Here, we consider that the femto BSs can exist anywhere from 0 to $\infty$. The LTs of the $I_{R_{1}}, I_{R}$ and $I_{r}$ for the blackout mode are expressed in the lemma below. 


$$
\begin{aligned}
\mathcal{C}_{m \prime(\overline{b k})}^{(M S)}=\int_{0}^{\infty} \int_{0}^{R_{1}\left(\frac{\beta_{21} P_{2}}{P_{1}}\right)^{1 / \eta}} \frac{4 \pi^{2} \lambda_{1} \lambda_{2} r_{1} R_{1}}{\left(1+\frac{T P_{2} r_{1}^{-\eta}}{P_{1} R_{1}^{-\eta}}\right) A_{f(\bar{b})}^{(M S)}} \exp \left(-\pi \lambda_{2} r_{1}^{2}-\pi \lambda_{1} R_{1}^{2}-\frac{2 \pi T}{(\eta-2)}\left\{\frac{\lambda_{2} P_{2} T}{P_{1} R_{1}^{-\eta}} r_{1}^{2-\eta} .\right.\right. \\
\left.\left.{ }_{2} F_{1}\left(1,1-\frac{2}{\eta}, 2-\frac{2}{\eta}, \frac{-P_{2} T r_{1}^{-\eta}}{P_{1} R_{1}^{-\eta}}\right)+\lambda_{1} T R_{12}^{2} F_{1}\left(1,1-\frac{2}{\eta}, 2-\frac{2}{\eta},-T\right)\right\}\right) d r_{1} d R_{1} .
\end{aligned}
$$

Lemma 9 (LTs of the interference in MS case): The LT of $I_{R_{1}}$ in the blackout mode with cooperative service from the second and third strongest macro BSs is given by

$$
\mathscr{L}_{I_{R_{1}}}(s)=\int_{0}^{R_{2}} \frac{2 R_{1}}{R_{2}^{2}\left(1+s P_{1} R_{1}^{-\eta}\right)} d R_{1} .
$$

The LT of $I_{R}$ in the blackout mode with BS cooperation can be expressed in terms of the hypergeometric function as

$\mathscr{L}_{I_{R}}(s)=\exp \left(\frac{-\pi \lambda_{1} s P_{1} R_{3}^{2-\eta}}{\eta-2}{ }_{2} F_{1}\left(1,1-\frac{2}{\eta}, 2-\frac{2}{\eta}, \frac{-s P_{1}}{R_{3}^{\eta}}\right)\right)$.

The LT of $I_{r}$ in the blackout case is given by

$$
\mathscr{L}_{I_{r}}(s)=\exp \left(-2 \pi^{2} \lambda_{2} \frac{\left(s P_{2}\right)^{2 / \eta}}{\eta} \csc \left(\frac{2 \pi}{\eta}\right)\right) .
$$

Proof: The LT of $I_{R_{1}}$ is obtained using the same procedure as done for $I_{r_{1}}$ in (42) but considering $s=\frac{T}{P_{1}\left(R_{2}^{-\eta}+R_{3}^{-\eta}\right)}$, interference region from 0 to $R_{2}$ and the conditional distribution obtained in (50). The LT of $I_{R}$ is obtained in the similar way as $\mathscr{L}_{I_{R}}(s)$ for $\underline{\text { FD }}$ case but with $s$ mentioned above and the interference boundary from $R_{3}$ to $\infty$. For $\mathscr{L}_{I_{r}}(s)$, we follow the same procedure as of $\mathscr{L}_{I_{R}}(s)$ with femto interference limits from 0 to $\infty$.

The above LTs evaluated at $\eta=4$ are boiled down to closed form expressions as given by

$$
\begin{gathered}
\left.\mathscr{L}_{I_{R_{1}}}(s)\right|_{\eta=4}=1-\sqrt{\frac{T}{1+R_{2}^{4} R_{3}^{-4}}} \arctan \left(\sqrt{\frac{1+R_{2}^{4} R_{3}^{-4}}{T}}\right) . \\
\left.\mathscr{L}_{I_{R}}(s)\right|_{\eta=4}=\exp \left(-\pi \lambda_{1} \sqrt{\frac{T}{R_{2}^{-4}+R_{3}^{-4}}} \arctan \left(\sqrt{\frac{T R_{2}^{4}}{R_{2}^{4}+R_{3}^{4}}}\right)\right) . \\
\left.\mathscr{L}_{I_{r}}(s)\right|_{\eta=4}=\exp \left(-\frac{\pi^{2} \lambda_{2}}{2} \sqrt{\frac{T P_{2}}{P_{1}\left(R_{2}^{-4}+R_{3}^{-4}\right)}}\right) .
\end{gathered}
$$

Using the service distance distribution and the LTs in Lemmas 8 and 9, we obtain the coverage probability for the $\underline{\mathrm{MS}}$ case as shown in the following theorem.

Theorem 7 (Coverage probability in MS case):

Considering two independent PPPs based two tier downlink cellular network with BS intensity $\lambda_{i}$ in a Rayleigh fading environment, the coverage probability for the blackout user in $\underline{\text { MS }}$ case with BS cooperation is given in (65).

Proof: We obtain the coverage probability for the blackout user with cooperation by substituting the LTs found in Lemma 9 in the conditional coverage probability expression given in (58) and integrating it over the service distance distribution obtained in Lemma 8.
The coverage probability for the blackout user with interference cancellation capabilities is given by the following theorem.

Theorem 8 (Coverage probability in MS case with IC): Considering two independent PPPs based two tier downlink cellular network with BS intensity $\lambda_{i}$ in a Rayleigh fading environment, the coverage probability for blackout users in the $\underline{\mathrm{MS}}$ case with interference cancellation capabilities is given in (66).

Proof: The theorem is obtained using the same methodology for obtaining Theorem 7 but with eliminating $I_{R_{1}}$ from (58).

Figs. 2(a) and 2(b) show the analysis and simulations for the coverage probabilities for all of the considered HO schemes without and with nearest BS interference cancellation. While the analysis is for stationary PPPs, the simulations in Figs. 2(a) and 2(b) account for user mobility. Consequently, the good match between the analysis and simulations validates our model. Fig. 2(a) shows the cost of HO skipping from the coverage probability perspective. That is, sacrificing the best SINR connectivity degrades the coverage probabilities even with BS cooperation. Such coverage probability degradation can be mitigated via IC as shown in Fig. 2(b). For instance, employing BS cooperation and IC, the coverage probability for the FS scheme is almost similar to the BS scheme. Although the proposed HO schemes degrade the coverage probability, they offer tangible improvements to the average throughput due to decrease in the the $\mathrm{HO}$ rate as shown in the next sections.

\section{HANDOVER COST}

In this section, we encompass user mobility effect and compute handover rates and cost for each $\mathrm{HO}$ skipping scheme. We define $\mathrm{HO}$ cost $D_{H O}$ as the normalized average time wasted during the execution of HOs. While handover delay is for each handoff, the handover cost averages and normalizes the handover delays over the entire user trajectory. Thus, $D_{H O}$ is the fraction of time where no data (i.e., control only) is transmitted to the test user. Note that the HO cost is different for each $\mathrm{HO}$ scheme due to different employed skipping strategies. Let $d_{i j}$ be the delay per $i$ to $j$ handover and $H_{i j}$ be the number of HOs from tier $i$ to $j$ per unit time, then $D_{H O}$ can be expressed as

$$
D_{H O}=\sum_{i}^{K} \sum_{j}^{K} H_{i j} * d_{i j} .
$$

where $K$ is the number of network tiers, which is 2 in our case. Also, we use $d_{m}$ and $d_{f}$ to denote macro-to-macro HO 


$$
\begin{aligned}
\mathcal{C}_{m, m(b k)}^{(M S)}=\int_{0}^{\infty} \int_{R_{2}}^{\infty} 4\left(\pi \lambda_{1}\right)^{3} R_{2}^{3} R_{3} \int_{0}^{R_{2}} \frac{1}{1+\frac{T R_{1}^{-\eta}}{R_{2}^{-\eta}+R_{3}^{-\eta}}} \frac{2 R_{1}}{R_{2}^{2}} d R_{1} \cdot \exp \left(-\pi \lambda_{1} R_{3}^{2}-\frac{\pi \lambda_{1} T R_{3}^{2-\eta}}{(\eta-2)\left(R_{2}^{-\eta}+R_{3}^{-\eta}\right)}\right. \\
\left.{ }_{2} F_{1}\left(1,1-\frac{2}{\eta}, 2-\frac{2}{\eta},-\frac{T R_{3}^{-\eta}}{R_{2}^{-\eta}+R_{3}^{-\eta}}\right)-\frac{2 \pi^{2} \lambda_{2}}{\eta}\left(\frac{T P_{2}}{R_{2}^{-\eta}+R_{3}^{-\eta}}\right)^{2 / \eta} \csc \left(\frac{2 \pi}{\eta}\right)\right) d R_{3} d R_{2} d R_{1} .
\end{aligned}
$$

$$
\begin{array}{r}
\mathcal{C}_{m, m(b k, I C)}^{(M S)}=\int_{0}^{\infty} \int_{R_{2}}^{\infty} 4\left(\pi \lambda_{1}\right)^{3} R_{2}^{3} R_{3} \exp \left(-\pi \lambda_{1} R_{3}^{2}-{ }_{2} F_{1}\left(1,1-\frac{2}{\eta}, 2-\frac{2}{\eta},-\frac{T R_{3}^{-\eta}}{R_{2}^{-\eta}+R_{3}^{-\eta}}\right)\right. \\
\left.\frac{\pi \lambda_{1} T R_{3}^{2-\eta}}{(\eta-2)\left(R_{2}^{-\eta}+R_{3}^{-\eta}\right)}-\frac{2 \pi^{2} \lambda_{2}}{\eta}\left(\frac{T P_{2}}{R_{2}^{-\eta}+R_{3}^{-\eta}}\right)^{2 / \eta} \csc \left(\frac{2 \pi}{\eta}\right)\right) d R_{3} d R_{2} .
\end{array}
$$

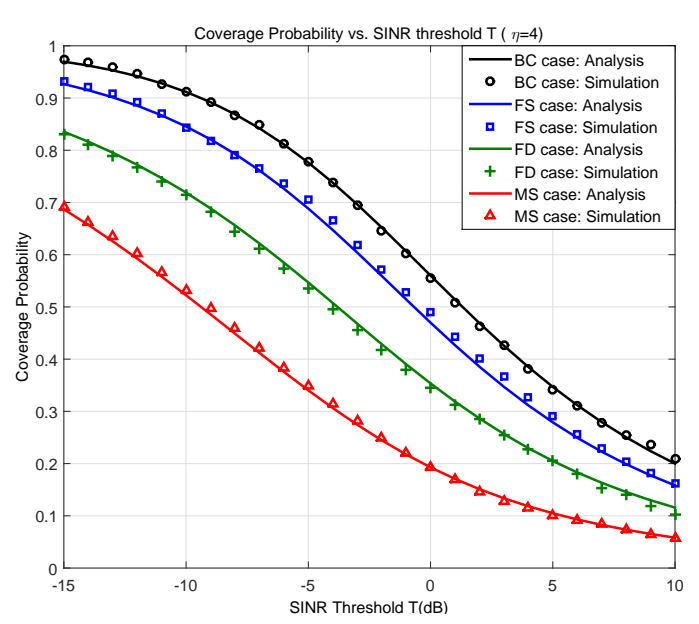

(a) Without Interference Cancellation.

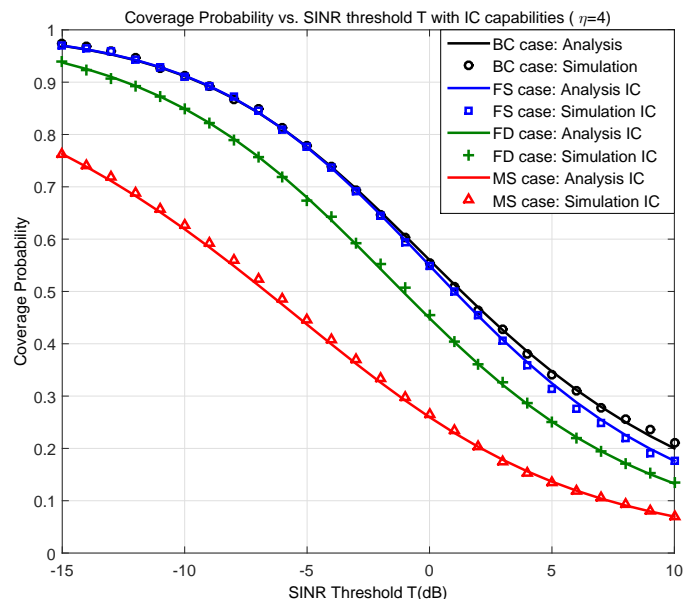

(b) With Interference Cancellation.

Fig. 2: Coverage probability plots for all cases at $\eta=4, \beta_{12}=0 \mathrm{~dB}$, transmission power $P_{1}=1$ watt, $P_{2}=0.1 P_{1}$ watt and $\mathrm{BS}$ intensities $\lambda_{1}=30 \mathrm{BS} / \mathrm{km}^{2}, \lambda_{2}=70 \mathrm{BS} / \mathrm{km}^{2}$.

delay and all femto related $\mathrm{HO}$ delays, respectively ${ }^{4}$. The $\mathrm{HO}$ rate is defined as the number of intersections between the user trajectory and the cell boundaries per unit time. Following [11], the tier $i$ to tier $j \mathrm{HO}$ rate is given by

$$
H_{i j}= \begin{cases}\frac{v}{\pi} L_{i j} & \text { if } i \neq j, \\ \frac{2 v}{\pi} L_{i j} & \text { if } i=j .\end{cases}
$$

where $v$ is the user velocity and $L_{i j}$ denotes the number of voronoi cell boundaries between a tier $i$ and tier $j$ BSs per unit length, which is given by

$$
L_{i j}= \begin{cases}\frac{\lambda_{i} \lambda_{j} F\left(x_{i j}\right)}{2\left(\sum_{n=1}^{K} \lambda_{n} x_{n k}^{2}\right)^{\frac{3}{2}}}+\frac{\lambda_{i} \lambda_{j} F\left(x_{j i}\right)}{2\left(\sum_{n=1}^{K} \lambda_{n} x_{n j}^{2}\right)^{\frac{3}{2}}} & \text { if } i \neq j, \\ \frac{\lambda_{i}^{2} F(1)}{2\left(\sum_{n=1}^{K} \lambda_{n} x_{n k}^{2}\right)^{\frac{3}{2}}} & \text { if } i=j .\end{cases}
$$

where $x_{11}=x_{22}=1, x_{12}=\left(\beta_{12} \frac{P_{1}}{P_{2}}\right)^{1 / \eta}, x_{21}=\frac{1}{x_{x_{12}}}$

$$
F(x)=\frac{1}{x^{2}} \int_{0}^{\pi} \sqrt{\left(x^{2}+1\right)-2 x \cos (\theta)} d \theta .
$$

In the $\mathrm{BC}$ scheme, the user experiences all types of HOs i.e. horizontal and vertical HOs. Thus, the total $\mathrm{HO}$ cost in $\underline{\mathrm{BC}}$

\footnotetext{
${ }^{4}$ We assume that $d_{m} \leq d_{f}$ because macro BSs usually have high speed dedicated (e.g., fiber-optic) connectivity to the core network. On the other hand, femto BSs may reach the core network via the macro BS through additional backhaul hop or via a shared ADSL/IP connectivity.
}

case is given by

$$
D_{H O}^{(B C)}=H_{11} d_{m}+\left(H_{12}+H_{21}+H_{22}\right) d_{f} .
$$

In FS scheme, the user skips every other femto BS and associates to all macro BSs. Therefore, the $\mathrm{HO}$ rate from femto-to-femto and from macro-to-femto is reduced to half. Thus, we can write $D_{H O}$ for $\underline{\mathrm{FS}}$ case as

$$
D_{H O}^{(F S)}=H_{11} d_{m}+\frac{H_{12}+H_{21}+H_{22}}{2} d_{f} .
$$

The user in the FD scheme skips all the femto BSs and associate to all macro BSs. Thus, $D_{H O}$ can be written as

$$
D_{H O}^{(F D)}=H_{11} d_{m}
$$

In $\underline{\text { MS }}$ case, the user disregards all femto BSs and skips every other macro BS. That is, the user spends $50 \%$ time in macro best connected phase and rest of the $50 \%$ in the macro blackout phase. Hence, we can write $D_{H O}$ as

$$
D_{H O}^{(M S)}=\frac{H_{11}}{2} d_{m}
$$

Figs. 3(a) and 3(b) show the HO cost for each HO skipping strategy with different BS intensities. It can be observed that the $\mathrm{HO}$ cost increases with the increase in the user velocity and the BS intensities. 


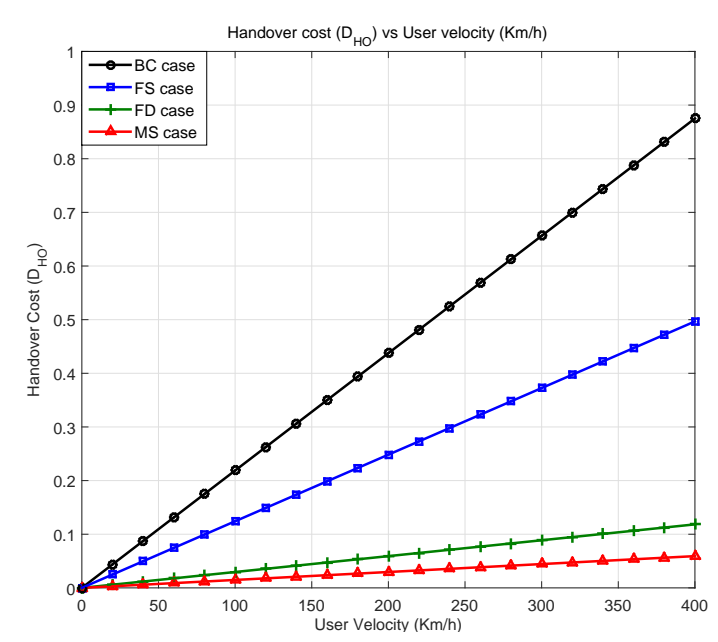

(a) $\lambda_{1}=30, \lambda_{2}=50 \mathrm{BS} / \mathrm{km}^{2}$.

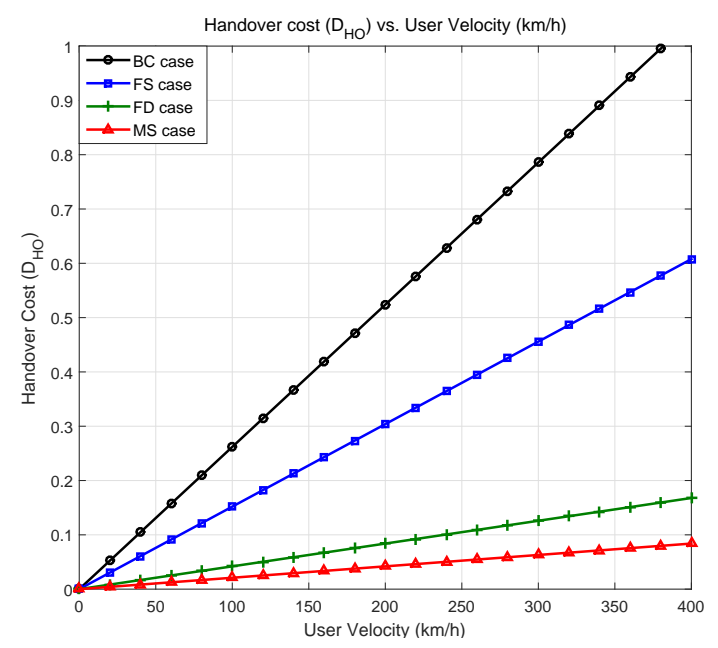

(b) $\lambda_{1}=50, \lambda_{2}=100 \mathrm{BS} / \mathrm{km}^{2}$.

Fig. 3: Handover cost vs. User velocity $(\mathrm{km} / \mathrm{h})$ with $P_{1}=1$ watt, $P_{2}=0.1 P_{1}$ watt, $\beta_{12}=0 \mathrm{~dB}, d_{m}=0.35 \mathrm{~s}, d_{f}=2 d_{m}$.

\section{USER THROUGHPUT}

In this section, we derive an expression for the user throughput, which is applicable to all HO skipping cases. In order to calculate the throughput, we need to omit the $\mathrm{HO}$ execution period. Thus the average throughput (AT) can be expressed as

$$
A T=W \mathcal{R}\left(1-D_{H O}\right) .
$$

where $W$ is the overall bandwidth of the channel and $\mathcal{R}$ is the achievable rate per unit bandwidth (i.e., nats/sec/Hz), which can be expressed as

$$
\mathcal{R}=\ln (1+T) \mathbb{P}[S I N R>T] .
$$

By performing the numerical evaluation for achievable rate per unit bandwidth in each case, we get $\mathcal{R}$ in nats $/ \mathrm{sec} / \mathrm{Hz}$ as shown in table II.

TABLE II: Achievable rate for all cases in nats $/ \mathrm{sec} / \mathrm{Hz}(T=6$ $\left.\mathrm{dB}, \beta_{12}=0 \mathrm{~dB}\right)$

\begin{tabular}{||ccc||}
\hline \multicolumn{3}{|c||}{ Achievable rate (nats/sec/Hz) } \\
\hline Case & Non-IC & IC \\
\hline \hline Best Connected $\mathcal{R}^{(B C)}$ & 0.50 & - \\
\hline Femto Skipping $\mathcal{R}^{(F S)}$ & 0.40 & 0.46 \\
\hline Femto Disregard $\mathcal{R}^{(F D)}$ & 0.29 & 0.36 \\
\hline Macro Skipping $\mathcal{R}^{(M S)}$ & 0.15 & 0.20 \\
\hline
\end{tabular}

\section{A. Design Insights}

We first study the negative impact of $\mathrm{HO}$ on the user's average throughput and highlight the velocity ranges over which the proposed $\mathrm{HO}$ schemes outperform the legacy best connected HO strategy. Fig. 4 shows a linear decay in the user throughput with velocity, which can be mitigated by the proposed HO skipping schemes. Similar performance behavior can be observed for highest SINR association in Figs. 4(a) and 4(b) as well as for biassed SINR association in Figs. 4(c) and $4(\mathrm{~d})$. Nevertheless, the gains provided by the proposed HO schemes are higher and occur at lower speeds for the case of biased association. This is because small cell edge users intrinsically experience blackout due to biasing, since the interfering macro BS signal is higher than the intended small BS signal. In all cases, the effective velocity ranges for each HO strategy is determined by the intersection points between the average throughput curves. For instance, once the user velocity exceeds $100 \mathrm{~km} / \mathrm{h}$, the femto skipping (FS) strategy provides more than $11 \%$ gains in the average throughput without biasing and $20 \%$ gains with biasing as compared to the best connected associations. Furthermore, the proposed adaptive HO skipping results show up to $77 \%$ gains in the average throughput as compared to the best connected association for the user velocity ranging from $80 \mathrm{~km} / \mathrm{h}$ to $200 \mathrm{~km} / \mathrm{h}$. However, it is worth noting that the cases $\underline{F S}$ and FD show gains in the average throughput at medium and high velocity ranges, respectively. Also, we can observe that the skipping of macros in a two tier network outperforms the RSS based association at very high user velocities.

To see the overall gain of network densification, we consider its positive and negative impacts on user throughput. From one side, increasing the BS intensity decreases the number of users associated to each BS, which increases the share each user gets from the BS resources. From the other side, increasing the BS intensity aggravates the handover cost. This tradeoff is highlighted in Fig. 5 by plotting the average throughput versus the BS intensity. The results in Fig. 5 are obtained by assuming stationary users modeled via a homogeneous PPP of intensity $\lambda_{u}$, utilizing the model in [36, Corollary 2] to evaluate the average number of users served by each BS, and [15, Equation 12] to calculate the average throughput per user assuming equal sharing among the users (including the test mobile user) for the resources in each BS. The figure shows that there is a turning point at which the handover cost dominates the performance such that densification degrades the user throughput. The figure also highlights the potential of FS HO strategy to extend the densification gain to higher BS intensities and user velocities. The FS HO scheme is particularly selected for the comparison in Fig. 5 because it outperforms the other schemes at the considered low user 


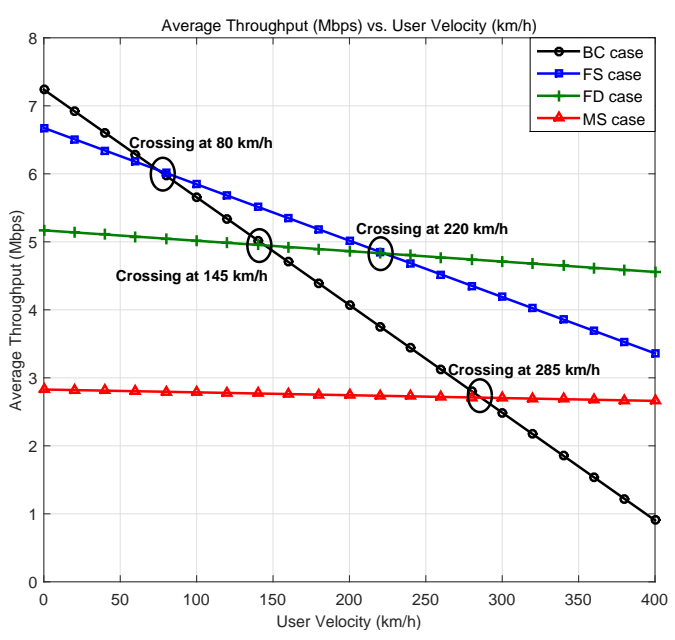

(a) $\beta_{21}=0 \mathrm{~dB}, d_{m}=0.35 \mathrm{~s}, d_{f}=2 d_{m}$.

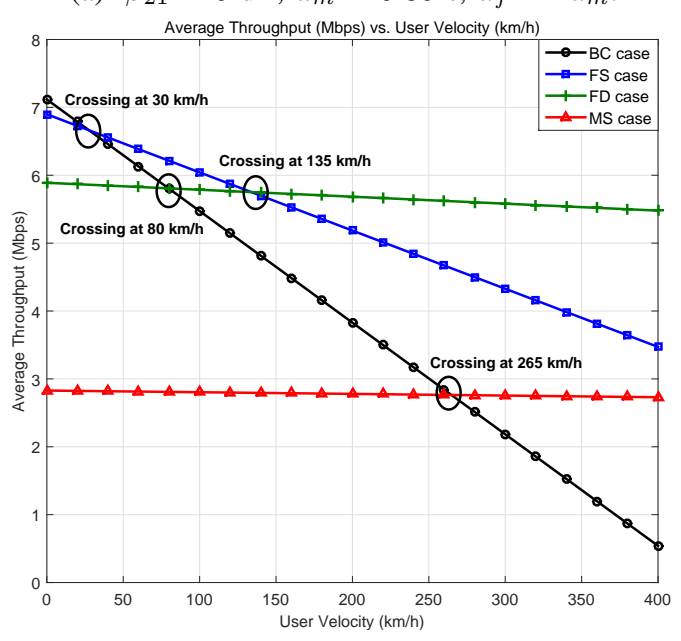

(c) $\beta_{21}=6 \mathrm{~dB}, d_{m}=0.35 \mathrm{~s}, d_{f}=2 d_{m}$.

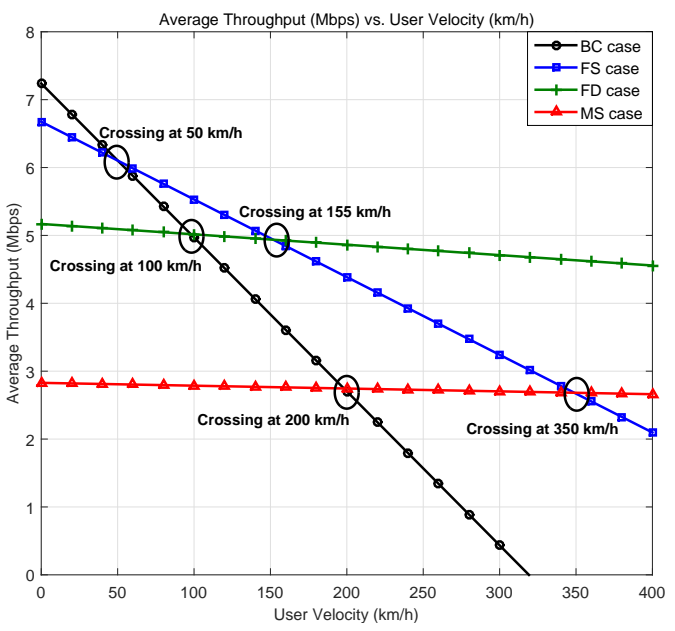

(b) $\beta_{21}=0 \mathrm{~dB}, d_{m}=0.35 \mathrm{~s}, d_{f}=3 d_{m}$.

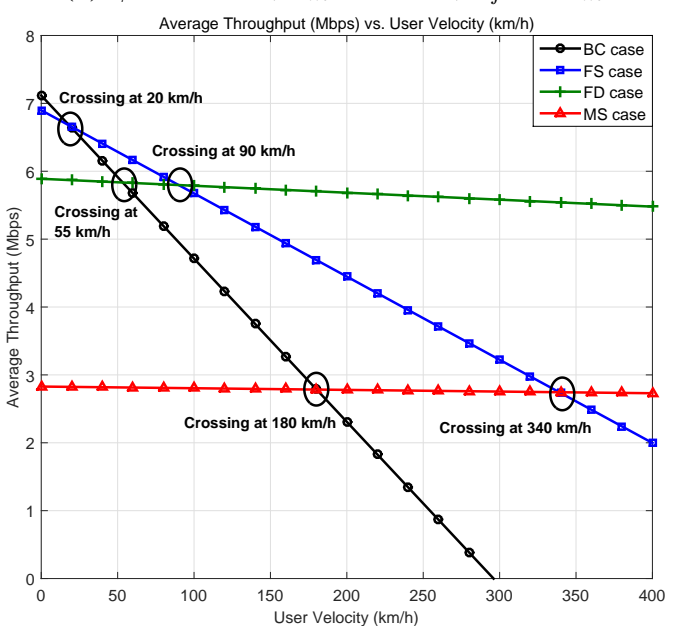

(d) $\beta_{21}=6 \mathrm{~dB}, d_{m}=0.35 \mathrm{~s}, d_{f}=3 d_{m}$.

Fig. 4: Average Throughput (Mbps) vs. User velocity $(\mathrm{km} / \mathrm{h})$ with $W=10 \mathrm{MHz}, P_{1}=1$ watt, $P_{2}=0.1 P_{1}$ watt, $\lambda_{1}=30$ $\mathrm{BS} / \mathrm{km}^{2}, \lambda_{2}=70 \mathrm{BS} / \mathrm{km}^{2}, T=6 \mathrm{~dB}$.

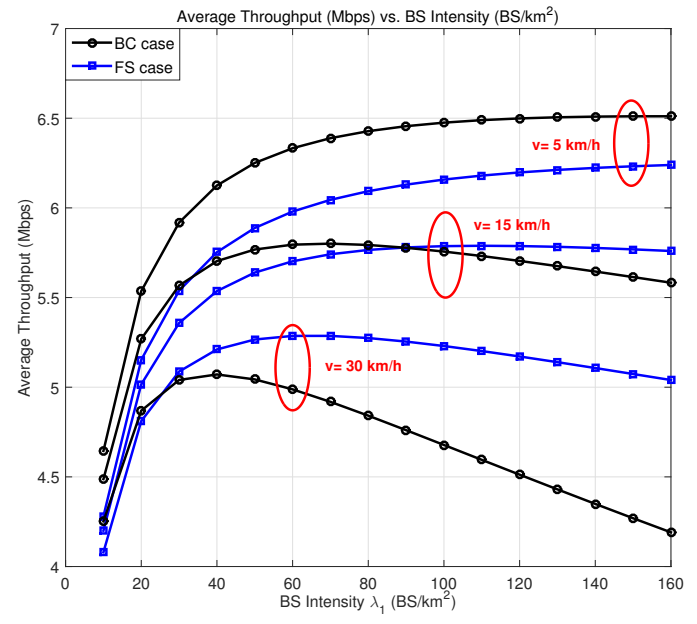

Fig. 5: Average throughput per user vs. BS intensity with $P_{1}=$ 1 watt, $P_{2}=0.1 P_{1}$ watt, $\lambda_{2}=2 \lambda_{1}, \lambda_{u}=10$ user $/ \mathrm{km}^{2}, T=6$ $\mathrm{dB}, \beta_{21}=0 \mathrm{~dB}, d_{m}=0.7 \mathrm{~s}, d_{f}=3 d_{m}$. velocity (cf. Fig. 4).

\section{CONCLUSION}

This paper proposes user velocity aware HO skipping schemes for two tier cellular network to enhance the average rate for mobile users. We develop an analytical paradigm to model the performance of the proposed cooperative HO skipping schemes in order to study the effect of HO delay on the user rate. The developed mathematical model is based on stochastic geometry and is validated via Monte Carlo simulations. The results manifest the negative impact of $\mathrm{HO}$ on the users' rate in dense cellular networks and emphasize the potential of the proposed $\mathrm{HO}$ schemes to mitigate such negative HO impact. Particularly, the results show up to $77 \%$ more rate gains, which can be harvested via the proposed $\mathrm{HO}$ schemes when compared to the conventional HO scheme that always maintains the best RSS association. For future work, we will extend our study towards location aware HO skipping. Thus, we will propose HO skipping based on user trajectory, which will maximize the gains while meeting the quality of service requirements. 


\section{APPENDIX}

\section{A. Proof of Lemma 2}

The Laplace transform of $I_{R}$ can be expressed as

$$
\mathscr{L}_{I_{R}(m)}(s)=\mathrm{E}\left[e^{-s I_{R}}\right]=\mathrm{E}\left[e^{-s \sum_{i \epsilon \phi_{1} \backslash b_{1}} P_{1} h_{i} R_{i}^{-\eta}}\right] .
$$

Due to the independence between fading coefficients and BSs locations, we get

$$
\begin{aligned}
\mathscr{L}_{I_{R}(m)}(s) & =\mathrm{E}_{\phi}\left\{\prod_{i \in \phi_{1} \backslash b_{1}} \mathrm{E}_{h_{i}}\left\{e^{-s P_{1} h_{i} R_{i}^{-\eta}}\right\}\right\} \\
& =\mathrm{E}_{\phi}\left\{\prod_{i \epsilon \phi_{1} \backslash b_{1}} \mathscr{L}_{h_{i}}\left(s P_{1} R_{i}^{-\eta}\right)\right\} .
\end{aligned}
$$

However, since $h_{i} \sim \exp (1)$, we can write

$$
\mathscr{L}_{I_{R}(m)}(s)=\mathrm{E}_{\phi}\left\{\prod_{i \in \phi_{1} \backslash b_{1}} \frac{1}{1+s P_{1} R_{i}^{-\eta}}\right\} .
$$

Using the probability generating functional (PGFL) for PPP [37] yields

$$
\mathscr{L}_{I_{R}(m)}(s)=\exp \left(-2 \pi \lambda_{1} \int_{R_{1}}^{\infty}\left(1-\frac{1}{1+s P_{1} v^{-\eta}}\right) v d v\right) .
$$

Now, invoke the change of variables $w=\left(s P_{1}\right)^{-1 / \eta} v$ and set $s=\frac{T R_{1}^{\eta}}{P_{1}}$ to get

$$
\begin{aligned}
\mathscr{L}_{I_{R}(m)}(s) & =\exp \left(-2 \pi \lambda_{1} R_{1}^{2} T^{2 / \eta} \int_{T^{-1 / \eta}}^{\infty} \frac{w}{1+w^{\eta}} d w\right), \\
& =\exp \left(-\frac{2 \pi \lambda_{1} T R_{1}^{2}}{\eta-2}{ }_{2} F_{1}\left(1,1-\frac{2}{\eta}, 2-\frac{2}{\eta},-T\right)\right) .
\end{aligned}
$$

The LT of $I_{r}$ can be written as

$$
\mathscr{L}_{I_{r}(m)}(s)=\mathrm{E}\left\{e^{-s \sum_{i \epsilon \phi_{2}} P_{2} h_{i} r_{i}^{-\eta}}\right\} .
$$

Following the same procedure utilized for $\mathscr{L}_{I_{R}(m)}(s)$ above and considering the interference region of femto BSs from $R_{1}\left(\frac{\beta_{21} P_{2}}{P 1}\right)^{1 / \eta}$ to $\infty$, we get the following expression for $\mathscr{L}_{I_{r}(m)}(s)$

$$
\begin{aligned}
\mathscr{L}_{I_{r}(m)}(s)=\exp \left(\frac{-2 \pi \lambda_{2} T R_{1}^{2}}{\eta-2}\left(\frac{\beta_{21} P_{2}}{P_{1}}\right)^{2 / \eta} \beta_{12}^{\eta} .\right. \\
\left.{ }_{2} F_{1}\left(1,1-\frac{2}{\eta}, 2-\frac{2}{\eta},-T \beta_{12}\right)\right) .
\end{aligned}
$$

Similarly, the LTs $\mathscr{L}_{I_{R(f)}}(s)$ and $\mathscr{L}_{I_{r(f)}}(s)$ in the femto association can be obtained using the macro interference region from $r_{1}\left(\frac{\beta_{12} P_{1}}{P 2}\right)^{1 / \eta}$ to $\infty$ and femto interference from $r_{1} \rightarrow \infty$.

\section{B. Proof of Lemma 3}

First, we write intensity measure of the points inside a ball $\mathbf{B}$ of radius $r$ as $\Lambda(\mathbf{B})=\pi \lambda r^{2}$ and the intensity function, which is given by $\lambda(x)=2 \pi \lambda r$. Then, using mapping theorem, we can write the intensity measure on a line from 0 to $y$ as $\Lambda([0, y])=\pi \lambda(P B y)^{2 / \eta}$ and the intensity function $\lambda(y)=\frac{2}{\eta} \pi \lambda(P B)^{2 / \eta} y^{2 / \eta-1}$. Now, using the superposition of point processes (eq. 5.19 in [37]), we can express the total intensity as

$$
\lambda(y)=\frac{2 \pi}{\eta}\left(\lambda_{1}\left(B_{1} P_{1}\right)^{2 / \eta}+\lambda_{2}\left(B_{2} P_{2}\right)^{2 / \eta}\right) y^{2 / \eta-1} .
$$

The conditional distance distribution of $r_{1}$ conditioned on the second strongest BS distance $x$ given in (21) is obtained as follows

$$
f_{r}\left(r_{1} \mid x\right)=\frac{\lambda\left(r_{1}\right)}{\int_{0}^{x} \lambda(z) d z}=\frac{2 r_{1}^{2 / \eta-1}}{\eta x^{2 / \eta}} .
$$

Using the null probability of PPP and employing mapping theorem, we can express the service distance distribution in a single tier network as

$$
\begin{aligned}
f_{Y}(y) & =\frac{d}{d y}\left(1-e^{-\pi \lambda(P B y)^{2 / \eta}}\right) \\
& =\frac{2}{\eta} \pi \lambda(P B)^{2 / \eta} y^{2 / \eta-1} e^{-\pi \lambda(P B)^{2 / \eta}} y^{2 / \eta}
\end{aligned}
$$

Following (81) and using the total intensity measure, we can write the PDF of $r_{1}$ (i.e., distance between the user and the strongest femto BS) in a two tier network as

$$
f_{r_{1}}(r)=\frac{2}{\eta} \pi \lambda_{t} r^{2 / \eta-1} \exp \left(-\pi r^{2 / \eta} \lambda_{t}\right)
$$

where $\lambda_{t}=\lambda_{1}\left(B_{1} P_{1}\right)^{2 / \eta}+\lambda_{2}\left(B_{2} P_{2}\right)^{2 / \eta}$. We can write the conditional distance distribution of the third strongest BS conditioning on $r_{1}$ as

$$
\begin{aligned}
& P\left[x_{2}<y \mid r_{1}\right]=1-\exp \left(\int_{r_{1}}^{y} \frac{2 \pi \lambda_{t}}{\eta} r^{2 / \eta-1} d r\right)- \\
& \exp \left(\int_{r_{1}}^{y} \frac{2 \pi \lambda_{t} r^{2 / \eta-1}}{\eta} d r\right) \int_{r_{1}}^{y} \frac{2 \pi \lambda_{t} r^{2 / \eta-1}}{\eta / 1 !} d r .
\end{aligned}
$$

By differentiating the above equation w.r.t. $y$, we get

$$
f\left(y \mid r_{1}\right)=\frac{2}{\eta}\left(\pi \lambda_{t}\right)^{2} y^{2 / \eta-1}\left(y^{2 / \eta}-r_{1}^{2 / \eta}\right) e^{-\pi \lambda_{t}\left(y^{2 / \eta}-r_{1}^{2 / \eta}\right)} .
$$

The conditional distance distribution of the second strongest BS conditioning on $r_{1}$ can be calculated as

$$
f_{x_{1}}\left(x \mid r_{1}\right)=\frac{\lambda(x)}{\int_{r_{1}}^{y} \lambda(z) d z}=\frac{2 x^{2 / \eta-1}}{\eta\left(y^{2 / \eta}-r_{1}^{2 / \eta}\right)} .
$$

The product of (84) and (84) yields the joint conditional distribution given by

$f_{x_{1}, x_{2}}\left(x, y \mid r_{1}\right)=\left(\frac{2}{\eta} \pi \lambda_{t}\right)^{2}(x y)^{2 / \eta-1} \exp \left(-\pi \lambda_{t}\left(y^{2 / \eta}-r_{1}^{2 / \eta}\right)\right)$.

Using the law of conditional probability (i.e. $f(a, b \mid c)=$ $\left.\frac{f(a, b, c)}{f(c)}\right)$, we get the joint distribution $f_{x_{1}, x_{2}, r}\left(x, y, r_{1}\right)$ as

$$
f_{x_{1}, x_{2}, r}\left(x, y, r_{1}\right)=\left(\frac{2}{\eta} \pi \lambda_{t}\right)^{3}\left(x y r_{1}\right)^{2 / \eta-1} \exp \left(-\pi \lambda_{t} y^{2 / \eta}\right) .
$$

By integrating the above distribution w.r.t. $r_{1}$, from $0 \rightarrow x$, we get $f_{x_{1}, x_{2}}(x, y)$ as

$$
f_{x_{1}, x_{2}}(x, y)=\frac{4}{\eta^{2}}\left(\pi \lambda_{t}\right)^{3} x^{4 / \eta-1} y^{2 / \eta-1} \exp \left(-\pi \lambda_{t} y^{2 / \eta}\right) .
$$

\section{Proof of Lemma 5}

The LT of $I_{r_{1}}$ can be expressed as

$$
\mathscr{L}_{I_{r_{1}}}(s)=\mathrm{E}\left[e^{-s I_{r_{1}}}\right]=\mathrm{E}\left[e^{-s \frac{h_{1}}{r_{1}}}\right]
$$

Since $h \sim \exp (1)$, we can write $\mathscr{L}_{I_{r_{1}}}(s)$ as

$$
\mathscr{L}_{I_{r_{1}}}(s)=\mathrm{E}\left[\frac{1}{1+s / r_{1}}\right]=\int_{0}^{x} \frac{1}{1+s / r_{1}} f\left(r_{1}\right) d r_{1},
$$


Using (21) obtained in Lemma 4 and substituting $s=$ $\frac{T}{x^{-1}+y^{-1}}$, we can express $\mathscr{L}_{I_{r_{1}}}(s)$ as

$$
\mathscr{L}_{I_{r_{1}}}(s)=\int_{0}^{x} \frac{2 r_{1}^{2 / \eta-1}}{\eta x^{2 / \eta}\left(1+\frac{T}{r_{1}\left(x^{-1}+y^{-1}\right)}\right)} d r_{1} .
$$

Similarly, the LT of $I_{a g g}$ can be written as

$$
\mathscr{L}_{I_{\text {agg }}}(s)=\mathrm{E}\left\{e^{-s \sum_{i \epsilon \phi \backslash b_{1}} h_{i} / u_{i}}\right\} .
$$

Due to the independence of the fading coefficients and the BSs locations, the exponential fading distribution $h_{i} \sim \exp (1)$, and applying the we obtain PGFL for PPP, we get

$$
\mathscr{L}_{I_{\text {agg }}}(s)=\exp \left(-\frac{2 \pi \lambda_{t}}{\eta} \int_{y}^{\infty} \frac{z^{2 / \eta-1}}{1+z / s} d z\right) .
$$

Finally, setting $s=\frac{T}{x^{-1}+y^{-1}}$ and after some simplification, we obtain

$$
\begin{aligned}
& \mathscr{L}_{I_{\text {agg }}}(s)=\exp \left(\frac{-2 \pi \lambda_{t} T y^{2 / n-1}}{(\eta-2)\left(x^{-1}+y^{-1}\right)}\right. \\
& \left.{ }_{2} F_{1}\left(1,1-\frac{2}{\eta}, 2-\frac{2}{\eta}, \frac{-T}{x^{-1} y+1}\right)\right) .
\end{aligned}
$$

\section{REFERENCES}

[1] B. Romanous, N. Bitar, A. Imran, and H. Refai, "Network densification: Challenges and opportunities in enabling 5G," in 20th International Workshop on Computer Aided Modelling and Design of Communication Links and Networks (CAMAD), 2015, pp. 129-134.

[2] X. Ge, S. Tu, G. Mao, C.-X. Wang, and T. Han, "5G Ultra-Dense cellular networks," IEEE Wireless Communications, vol. 23, no. 1, pp. 72-79, 2016.

[3] H. Zhang, Y. Dong, J. Cheng, M. Hossain, V. Leung et al., "Fronthauling for $5 \mathrm{~g}$ lte-u ultra dense cloud small cell networks," IEEE Wireless Communications ,Accepted, 2016.

[4] H. Zhang, L. Hui, M. Wenmin, W. Zheng, W. Xiangming, and C. Jiang, "Mobility robustness optimization in femtocell networks based on ant colony algorithm," IEICE transactions on communications, vol. 95, no. 4, pp. 1455-1458, 2012.

[5] H. Zhang, W. Ma, W. Li, W. Zheng, X. Wen, and C. Jiang, "Signalling cost evaluation of handover management schemes in LTE-advanced femtocell,' in Vehicular Technology Conference (VTC Spring), 2011 IEEE 73rd. IEEE, 2011, pp. 1-5.

[6] H. Zhang, C. Jiang, J. Cheng, and V. C. Leung, "Cooperative interference mitigation and handover management for heterogeneous cloud small cell networks," IEEE Wireless Communications, vol. 22, no. 3, pp. 92-99, 2015.

[7] N. Sinclair, D. Harle, I. A. Glover, and R. C. Atkinson, "A kernel methods approach to reducing handover occurrences within lte," in European Wireless, 2012. EW. 18th European Wireless Conference. VDE, 2012, pp. 1-8.

[8] H. ElSawy, E. Hossain, and M. Haenggi, "Stochastic geometry for modeling, analysis, and design of multi-tier and cognitive cellular wireless networks: A survey," IEEE Commun. Surveys Tuts., vol. 15, no. 3, pp. 996-1019, 2013.

[9] H. ElSawy, A. Sultan-Salem, M. S. Alouini, and M. Z. Win, "Modeling and analysis of cellular networks using stochastic geometry: A tutorial," IEEE Commun. Surveys Tuts., accepted 2016.

[10] X. Lin, R. K. Ganti, P. J. Fleming, and J. G. Andrews, "Towards understanding the fundamentals of mobility in cellular networks," IEEE Trans. Wireless Commun., vol. 12, no. 4, pp. 1686-1698, 2013.

[11] W. Bao and B. Liang, "Stochastic geometric analysis of user mobility in heterogeneous wireless networks," IEEE J. Sel. Areas Commun., vol. 33, no. 10, pp. 2212-2225, Oct 2015.

[12] S. Sadr and R. Adve, "Handoff rate and coverage analysis in multi-tier heterogeneous networks," IEEE Trans. Wireless Commun., vol. 14, no. 5 , pp. $2626-2638,2015$.

[13] G. Zhang, T. Q. Quek, A. Huang, and H. Shan, "Delay and reliability tradeoffs in heterogeneous cellular networks," IEEE Trans. Wireless Commun., pp. 1101-1113, 2016.
[14] X. Ge, J. Ye, Y. Yang, and Q. Li, "User mobility evaluation for 5G small cell networks based on individual mobility model," IEEE J. Sel. Areas Commun., vol. 34, no. 3, pp. 528-541, 2016.

[15] H. Ibrahim, H. Elsawy, U. T. Nguyen, and M.-S. Alouini, "Mobilityaware modeling and analysis of dense cellular networks with C-plane/Uplane split architecture," IEEE Trans. Commun., 2016, Submitted, Available at http://arxiv.org/pdf/1607.06439v1.pdf.

[16] R. Arshad, H. Elsawy, S. Sorour, T. Y. Al-Naffouri, and M.-S. Alouini, "Handover management in dense cellular networks: A stochastic geometry approach," in IEEE International Conference on Communications (ICC), 2016.

[17] _ - "Cooperative handover management in dense cellular networks," in IEEE Global Communications Conference (GLOBECOM), 2016, available at http://arxiv.org/pdf/1607.03378.pdf.

[18] 3GPP TR 36.819 v11.0.0, "Coordinated multi-point operation for LTE,' 3GPP TSG RAN WG1, Tech. Rep., Sept. 2011.

[19] A. Davydov, G. Morozov, I. Bolotin, and A. Papathanassiou, "Evaluation of joint transmission CoMP in C-RAN based LTE-A hetnets with large coordination areas," in IEEE Globecom Workshops (GC Wkshps). IEEE, 2013, pp. 801-806.

[20] B. Cheng, X. Mi, X. Xu, Z. Xu, X. Xu, and M. Zhao, "A real-time implementation of CoMP transmission based on cloud-RAN infrastructure," in International Wireless Communications and Mobile Computing Conference (IWCMC), Aug 2014, pp. 1033-1038.

[21] J. G. Andrews, F. Baccelli, and R. K. Ganti, "A tractable approach to coverage and rate in cellular networks," IEEE Trans. Commun., vol. 59, no. 11, pp. 3122-3134, November 2011.

[22] A. Guo and M. Haenggi, "Spatial stochastic models and metrics for the structure of base stations in cellular networks," IEEE Trans. Wireless Commun., vol. 12, no. 11, pp. 5800-5812, November 2013.

[23] W. Lu and M. Di Renzo, "Stochastic geometry modeling of cellular networks: Analysis, simulation and experimental validation," in Proceedings of the 18th ACM International Conference on Modeling, Analysis and Simulation of Wireless and Mobile Systems, 2015, pp. 179-188.

[24] S. Parkvall, A. Furuskar, and E. Dahlman, "Evolution of LTE toward IMT-advanced," IEEE Communications Magazine, vol. 49, no. 2, pp. 84-91, 2011.

[25] H.-S. Jo, Y. J. Sang, P. Xia, and J. G. Andrews, "Heterogeneous cellular networks with flexible cell association: A comprehensive downlink SINR analysis," IEEE Trans. Wireless Commun., vol. 11, no. 10, pp. 3484 3495, 2012.

[26] H. ElSawy, E. Hossain, and S. Camorlinga, "Traffic offloading techniques in two-tier femtocell networks," in IEEE International Conf. on Commun. (ICC). IEEE, 2013, pp. 6086-6090.

[27] P. F. Ash and E. D. Bolker, "Generalized dirichlet tessellations," Ge ometriae Dedicata, vol. 20, no. 2, pp. 209-243, 1986.

[28] M. Thorpe, M. Kottkamp, A. Rössler, and J. Schütz, "LTE location based services technology introduction," Rohde \& Schwarz, 2013, available at http://cdn.rohde-schwarz.com/pws/dl_downloads/dl_common_library/ dl_brochures_and_datasheets/pdf_1/LTE_LBS_White_Paper.pdf.

[29] Y.-u. Chung and D. Cho, "Velocity estimation using adaptive array antennas," in IEEE Vehicular Technology Conference, vol. 4. IEEE; 1999, 2001, pp. 2565-2569.

[30] A. Merwaday, I. Güvenç, W. Saad, A. Mehbodniya, and F. Adachi, "Sojourn time-based velocity estimation in small cell poisson networks," IEEE Communications Letters, vol. 20, no. 2, pp. 340-343, 2016.

[31] J. Horng, G. Vannucci, and J. Zhang, "Down-link interference cancellation for high-data-rate channels in advanced digital wireless networks," Nov. 7 2002, US Patent App. 10/289,712”.

[32] T. Mahmoodi and S. Seetharaman, "On using a SDN-based control plane in 5G mobile networks," in Wireless World Research Forum, meeting, vol. 32. Citeseer, 2014.

[33] M. Abramowitz and I. A. Stegun, Handbook of Mathematical Functions, Tenth printing. $\quad$ Dover Publications, 1972.

[34] P. Madhusudhanan, J. G. Restrepo, Y. Liu, T. X. Brown, and K. R. Baker, "Downlink performance analysis for a generalized shotgun cellular system," IEEE Trans. Wireless Commun., vol. 13, no. 12, pp. 66846696, 2014.

[35] M. Haenggi, "On distances in uniformly random networks," IEEE Trans. Inf. Theory, vol. 51, no. 10, pp. 3584-3586, Oct. 2005.

[36] S. Singh and J. G. Andrews, "Joint resource partitioning and offloading in heterogeneous cellular networks," IEEE Trans. Wireless Commun., vol. 13, no. 2, pp. 888-901, February 2014.

[37] S. N. Chiu, D. Stoyan, W. Kendall, and J. Mecke, Stochastic Geometry and its Applications. John Wiley \& Sons, 2013. 


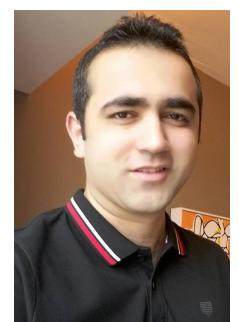

Rabe Arshad received the B.Sc. degree in electrical engineering (Gold Medalist) from University of Engineering \& Technology (UET) Lahore, Pakistan in 2010. Right after the graduation, he joined Mobilink, a multi-national cellular operator, as Radio Design and Traffic Engineer and served for four years till 2015. He received a Mobilink Century Club award in 2014 , for his exceptional contribution in various nationwide projects. During his stay in Mobilink, he received Masters degree in Project Management from Shaheed Zulfikar Ali Bhutto Institute of Science and Technology (SZABIST), Pakistan in 2014. He is currently working towards M.Sc degree in electrical engineering with the Department of Electrical Engineering, King Fahd University of Petroleum and Minerals (KFUPM), KSA. His research interests include stochastic geometry, handover management, point process theory and heterogeneous wireless networks.

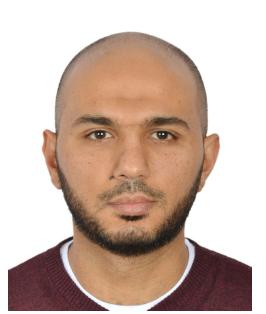

Hesham EISawy (S'10, M'14) received the B.Sc. degree in Electrical Engineering from Assiut University, Assiut, Egypt, in 2006, the M.Sc. degree in Electrical Engineering from Arab Academy for Science and Technology, Cairo, Egypt, in 2009, and the Ph.D. degree in Electrical Engineering from the University of Manitoba, Winnipeg, MB Canada, in 2014. Currently, he is a postdoctoral fellow with the Computer, Electrical, and Mathematical Sciences and Engineering Division, King Abdullah University of Science and Technology (KAUST), Saudi Arabia, and also has an adjunct affiliation to the school of Computer Science \& Engineering, York University, Canada. During the period of 2006-2010, he worked at the National Telecommunication Institute, Egypt, where he conducted professional training both at the national and international levels, as well as research on network planning. From 2010 to 2014, he worked with TRTech, Winnipeg, MB, Canada, as a Student Researcher. For his academic excellence, he has received several academic awards, including the NSERC Industrial Postgraduate Scholarship during the period of 2010-2013, and the TRTech Graduate Students Fellowship in the period of 2010-2014. He also received the best paper award in the ICC 2015 workshop on small cells and $5 \mathrm{G}$ networks. He is recognized as an exemplary reviewer by the IEEE Transactions of communication in 2015 \& 2016. His research interests include statistical modeling of wireless networks, stochastic geometry, and queueing analysis for wireless communication networks.

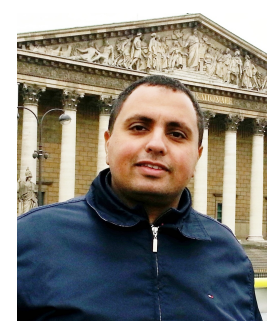

Sameh Sorour (S'98, M'11, SM'16) is an Assistant Professor at the Department of Electrical and Computer Engineering, University of Idaho. He received his B.Sc. and M.Sc. degrees in Electrical Engineering from Alexandria University, Egypt, in 2002 and 2006, respectively. In 2011, he obtained his $\mathrm{Ph} . \mathrm{D}$. degree in Electrical and Computer Engineering from University of Toronto, Canada. After two postdoctoral fellowships at University of Toronto and King Abduallah University of Science and Technology (KAUST), he joined King Fahd University of Petroleum and Minerals (KFUPM) in 2013 before moving to University of Idaho in 2016. His research interests include network coding, device-to-device networking, internet of things (IoT) and IoT-enabled systems, cloud and fog networking, mathematical modelling and optimization for smart systems.

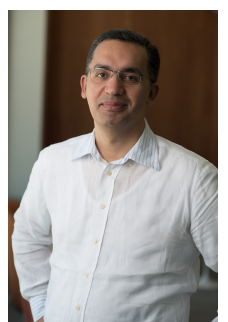

Tareq Al-Naffouri received the B.S. degrees in mathematics and electrical engineering (with first honors) from King Fahd University of Petroleum and Minerals, Dhahran, Saudi Arabia, the M.S. degree in electrical engineering from the Georgia Institute of Technology, Atlanta, in 1998, and the Ph.D. degree in electrical engineering from Stanford University, Stanford, CA, in 2004.

He was a visiting scholar at California Institute of Technology, Pasadena, CA, from January to August 2005 and during summer 2006. He was a Fulbright scholar at the University of Southern California from February to September 2008. He has held internship positions at NEC Research Labs, Tokyo, Japan, in 1998, Adaptive Systems Lab, University of California at Los Angeles in 1999, National Semiconductor, Santa Clara, CA, in 2001 and 2002, and Beceem Communications Santa Clara, CA, in 2004. He is currently an Associate Professor at the Electrical Engineering Department, King Abdullah University of Science and Technology (KAUST). His research interests lie in the areas of sparse, adaptive, and statistical signal processing and their applications and in network information theory. He has over 160 publications in journal and conference proceedings, 9 standard contributions, 10 issued patents, and 6 pending.

Dr. Al-Naffouri is the recipient of the IEEE Education Society Chapter Achievement Award in 2008 and Al-Marai Award for innovative research in communication in 2009. Dr. Al-Naffouri has also been serving as an Associate Editor of Transactions on Signal Processing since August 2013.

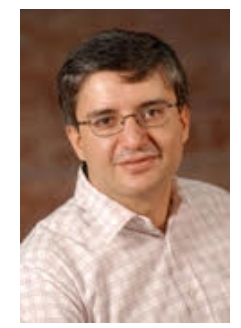

Mohamed-Slim Alouini (S'94, M'98, SM'03, F'09) was born in Tunis, Tunisia. He received the Ph.D. degree in Electrical Engineering from the California Institute of Technology (Caltech), Pasadena, CA, USA, in 1998. He served as a faculty member in the University of Minnesota, Minneapolis, MN, USA, then in the Texas A\&M University at Qatar, Education City, Doha, Qatar before joining King Abdullah University of Science and Technology (KAUST), Thuwal, Makkah Province, Saudi Arabia as a Professor of Electrical Engineering in 2009. His current research interests include the modeling, design, and performance analysis of wireless communication systems. 